\title{
Doubly-charged particles at the Large Hadron Collider
}

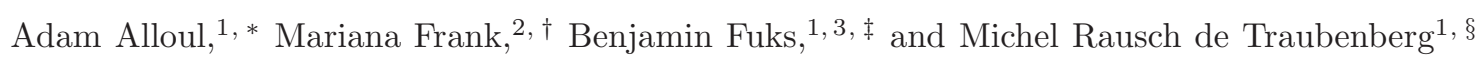 \\ ${ }^{1}$ Institut Pluridisciplinaire Hubert Curien/Département Recherches Subatomiques, \\ Université de Strasbourg/CNRS-IN2P3, 23 Rue du Loess, F-67037 Strasbourg, France \\ ${ }^{2}$ Department of Physics, Concordia University, 7141 Sherbrooke St. West, Montreal, Quebec, Canada H4B 1 R6 \\ ${ }^{3}$ Theory Division, Physics Department, CERN, CH-1211 Geneva 23, Switzerland
}

(Dated: September 3, 2018)

\begin{abstract}
In this work we investigate the production and signatures of doubly-charged particles at the Large Hadron Collider. We start with the Standard Model particle content and representations and add generic doubly-charged exotic particles. We classify these doubly-charged states according to their spin, considering scalar, fermionic and vectorial fields, and according to their $S U(2)_{L}$ representation, being chosen to be either trivial, fundamental, or adjoint. We write the most general interactions between them and the Standard Model sector and study their production modes and possible decay channels. We then probe how they can most likely be observed and how particles with different spin and $S U(2)_{L}$ representations could be possibly distinguished.
\end{abstract}

PACS numbers: 14.80.-j, 13.85.Rm

\section{INTRODUCTION}

The search for new particles has been given a boost with the discovery of a Higgs boson at the Large Hadron Collider (LHC) at CERN [1, 2]. While we are still awaiting confirmation of whether this is the long-awaited Higgs boson of the Standard Model (SM) or, as it appears at present, a Higgs boson with possibly tantalizing signs of new physics, phenomenologists are trying to predict benchmarks for beyond the Standard Model (BSM) physics that could follow. Some adhere to long-hyped scenarios such as weak-scale supersymmetry (either in its minimal reincarnation, or in a non-minimal form), extended gauge structures, extra-dimensional models, or Higgs composite models, and base their analyses on most likely and telling signatures of the models. Yet there has been also some interest in testing more generic collider features, that would have clear signatures and may be common to more models. The advantage is the fact that several models might yield the same collider signals, so that a simple but general model with minimal additions (particles and interactions) over the SM might be useful for obtaining results easy to compare with data. A small set of model parameters is usually involved, such as the masses of the new particles and the coupling strength of their interactions. In this setup, one could think of the SM as a limiting case when the new physics sector decouples.

Adopting this bottom-up approach for new physics phenomenology, the cleanest and clearest results are most likely obtained for exotic particles, i.e., particles with quantum numbers unlike those from the SM particle content. In this work, we propose to investigate the possibility for supplementing the Standard Model with a very simple kind of exotic states, which we denote by $X^{++}$and which are either scalar, fermion or vector fields with two units of electric charge. We keep our analysis as general as possible by allowing the new particles to belong to different $S U(2)_{L}$ representations, but further restrict ourselves to the simplest ones, namely the singlet, doublet and triplet cases. This goes along the same lines as several recent works investigating doubly-charged particles in more or less generic situations [3 8 ], our work being however the only analysis studying in detail the collider implications of various spin and weak isospin quantum numbers.

From a top-down point of view, it must be noted that such doubly-charged particles appear in many BSM scenarios, and thus are of particular interest to model builders. As examples, doubly-charged scalar states, often dubbed doublycharged Higgs fields, appear in left-right symmetric models [9 15] or in see-saw models for neutrino masses with Higgs

\footnotetext{
*Electronic address: adam.alloul@iphc.cnrs.fr

${ }^{\dagger}$ Electronic address: mfrank@alcor.concordia.ca

${ }^{\ddagger}$ Electronic address: fuks@cern.ch

$\S$ Electronic address: michel.rausch@iphc.cnrs.fr
} 
triplets [16 31]. Doubly-charged fermions can appear in extra-dimensional models including custodian taus 32 37], in new physics models inspired by string theories [38] or as the supersymmetric partners for the doubly-charged scalar fields in supersymmetric extensions of left-right symmetric models 39 42. Finally, BSM theories with an extended gauge group often include doubly-charged vector bosons [43 47] although it is also possible to consider vector states with a double electric charge independently of any gauge-group structure, as in models with a non-commutative geometry or in composite or technicolor theories [48 56].

Subsequently, same-sign dilepton and/or doubly-charged Higgs bosons resonances have been the topic of many accelerator analyses in the past. Usually assumed to be produced either singly or in pairs, no events have been observed by experiments around the Large Electron Positron collider (LEP) [57 60], the Hadron Electron Ring Accelerator (HERA) 61, 62 and the Tevatron 63 66. The most up-to-date bounds have however been more recently derived by the LHC experimental collaborations. Both ATLAS and CMS have searched for long-lived doubly-charged states [67, 68], basing their analyses either on identification of the new particles by using their longer time-of-flight to the outer subdetectors or on their anomalous energy loss along their tracks. Assuming a Drell-Yan-like pair production, the long-lived doubly-charged state masses have been constrained to lie above $685 \mathrm{GeV}$ after analyzing $5 \mathrm{fb}^{-1}$ of $\mathrm{LHC}^{-1}$ collisions at a center-of-mass energy of $\sqrt{S_{h}}=7 \mathrm{TeV}$ and $18.8 \mathrm{fb}^{-1}$ of collisions at $\sqrt{S_{h}}=8 \mathrm{TeV}[67$ ]. Nevertheless, these limits do not hold for promptly-decaying doubly-charged particles. In this case, dedicated studies only exist for doubly-charged Higgs bosons. Being pair-produced, they are then assumed to decay into a pair of leptons with the same electric charge through Majorana-type interactions [69, 70]. Assuming a branching fraction of $100 \%$ decays into leptons, i.e., neglecting the possible decays into a $W$-boson pair, the doubly-charged Higgs mass has been constrained to be larger than about $450 \mathrm{GeV}$. All these existing mass bounds can however be easily evaded by relaxing the rather constraining new physics assumptions. We hence follow the approach of most model-builders and consequently assume the new particle mass to be a free parameter.

Our work is organized as follows. In Section 2 we describe in detail our model for particles and interactions, differentiating in the discussion into spin and weak-isospin fields. We also compute analytical cross sections for the production of the new states at hadron colliders and for their decays into Standard Model particles. We then dedicate Section 3 to a detailed numerical analysis of doubly-charged particle signals at the LHC and briefly discuss, in Section 4. different kinematical variables that would allow for distinguishing the spin and/or $S U(2)_{L}$ quantum numbers of the new states. Finally, we summarize our results in Section 5.

\section{SIMPLIFIED MODELS FOR EXOTIC DOUBLY-CHARGED STATES}

Following the approach of the LHC New Physics Working Group [71], specific final state topologies are described by means of dedicated simplified models. They consist of minimal extensions of the Standard Model where the number of new states and operators is maximally reduced. Moreover, the model parameters are translated in terms of relevant products of cross sections and branching ratios so that LHC data can be easily reinterpreted in terms of constraints on these quantities. In this work, we construct a set of simplified models describing all the mechanisms yielding the production of doubly-charged particles, followed by their subsequent decays into pairs of charged leptons (possibly together with additional neutral states when relevant) with the same electric charges. Only final state signatures with three leptons or more will be considered, as the associated Standard Model background is known to be under good control.

We start with the Standard Model field content and the $S U(3)_{C} \times S U(2)_{L} \times U(1)_{Y}$ gauge group and then add an exotic doubly-charged, non-colored, state lying in a specific representation of the Lorentz group and $S U(2)_{L}$. Motivated by the most common existing new physics theories, we restrict ourselves to scalar, spin $1 / 2$ and vector states which we assume to lie either in the trivial, fundamental or adjoint representation of $S U(2)_{L}$. However, higher spin states or higher-dimensional representations of $S U(2)_{L}$ could be considered, such as in Ref. [56] where the phenomenology of excited leptons in the $\underset{\sim}{4}$ representation of $S U(2)_{L}$ is investigated. In addition, the hypercharge quantum numbers of the new multiplet are chosen so that the doubly-charged component is always the state with the highest electric charge. Finally, any interaction allowed by model symmetries but irrelevant for our study is omitted from the Lagrangians presented in this section.

In the rest of this section, we construct simplified models following a classification of the doubly-charged states by their spin. We focus on their signals at the LHC and analytically compute cross sections and decay widths relevant for the production of final states with three leptons or more, the corresponding numerical analysis being performed in Section 3. This will guide us to a choice of benchmark scenarios to be considered for a more advanced study based on Monte Carlo simulations, as in Section 4 


\subsection{Spin 0 doubly-charged particles}

In the following, we focus on simplified models describing the dynamics of $S U(2)_{L}$ multiplets containing, as a component with the highest electric charge, a doubly-charged state. We denote by $\phi, \Phi$ and $\boldsymbol{\Phi}$ three complex scalar fields lying in the $\underset{\sim}{\mathbf{1}}, \underset{\sim}{\mathbf{2}}$ and $\underset{\sim}{\mathbf{3}}$ representations of $S U(2)_{L}$, respectively, with hypercharge quantum numbers set to $Y_{\phi}=2, Y_{\Phi}=3 / 2, \widetilde{Y}_{\Phi}=1$,

$$
\phi \equiv \phi^{++}, \quad \Phi^{i} \equiv\left(\begin{array}{c}
\Phi^{++} \\
\Phi^{+}
\end{array}\right) \quad \text { and } \quad \boldsymbol{\Phi}_{j}^{i} \equiv\left(\begin{array}{cc}
\frac{\Phi^{+}}{\sqrt{2}} & \boldsymbol{\Phi}^{++} \\
\boldsymbol{\Phi}^{0} & -\frac{\boldsymbol{\Phi}^{+}}{\sqrt{2}}
\end{array}\right) .
$$

In the last expression, we have employed the matrix representation for triplet fields defined by

$$
\boldsymbol{\Phi}_{j}^{i}=\frac{1}{\sqrt{2}}\left(\sigma_{a}\right)^{i}{ }_{j} \boldsymbol{\Phi}^{a}
$$

the matrices $\sigma^{a}$ being the Pauli matrices and $a=1,2,3$ a $S U(2)_{L}$ adjoint gauge index ${ }^{1}$. Diagonalizing the third generator of $S U(2)_{L}$ in the adjoint representation, the gauge eigenstates $\boldsymbol{\Phi}^{a}$ can be linked to the physical masseigenstates $\boldsymbol{\Phi}^{0}, \boldsymbol{\Phi}^{+}$and $\boldsymbol{\Phi}^{++}$by means of

$$
\boldsymbol{\Phi}^{1}=\frac{1}{\sqrt{2}}\left[\boldsymbol{\Phi}^{0}+\boldsymbol{\Phi}^{++}\right], \quad \boldsymbol{\Phi}^{2}=\frac{1}{\sqrt{2} i}\left[\boldsymbol{\Phi}^{0}-\boldsymbol{\Phi}^{++}\right] \quad \text { and } \quad \boldsymbol{\Phi}^{3}=\boldsymbol{\Phi}^{+} .
$$

Kinetic and gauge interaction terms for the three fields of Eq. (2.1) are fixed by gauge invariance,

$$
\mathcal{L}_{\mathrm{kin}}=D_{\mu} \phi^{\dagger} D^{\mu} \phi+D_{\mu} \Phi_{i}^{\dagger} D^{\mu} \Phi^{i}+D_{\mu} \boldsymbol{\Phi}_{a}^{\dagger} D^{\mu} \boldsymbol{\Phi}^{a}+\ldots
$$

the covariant derivatives being given by

$$
\begin{aligned}
D_{\mu} \phi & =\partial_{\mu} \phi-2 i g^{\prime} B_{\mu} \phi \\
D_{\mu} \Phi^{i} & =\partial_{\mu} \Phi^{i}-\frac{3}{2} i g^{\prime} B_{\mu} \Phi^{i}-i g \frac{\left(\sigma_{a}\right)_{j}^{i}}{2} \Phi^{j} W_{\mu}^{a}, \\
D_{\mu} \boldsymbol{\Phi}^{a} & =\partial_{\mu} \boldsymbol{\Phi}^{a}-i g^{\prime} B_{\mu} \boldsymbol{\Phi}^{a}+g \varepsilon_{b c}{ }^{a} \boldsymbol{\Phi}^{c} W_{\mu}^{b},
\end{aligned}
$$

and the dots standing for mass terms. In the expressions above, $g$ and $g^{\prime}$ are the weak and hypercharge coupling constants, respectively, and we have normalized the structure constants of $S U(2)$ as $\varepsilon_{12}{ }^{3}=1$. We have also introduced the electroweak gauge bosons denoted by $B_{\mu}$ and $W_{\mu}^{a}$. The Lagrangian above also allows the components of fields lying in a non-trivial representation of $S U(2)_{L}$ to decay into each other, together with an accompanying gauge boson, if kinematically allowed. However, we choose to focus in this work on the low mass region of the parameter space so that splittings, induced, e.g., by electroweak symmetry breaking, are assumed smaller than the weak boson masses. In order to allow for the extra fields $\phi, \Phi$ and $\boldsymbol{\Phi}$ to decay, new Yukawa interactions are hence required,

$$
\mathcal{L}_{\mathrm{yuk}}=\frac{1}{2} y^{(1)} \phi \bar{l}_{R}^{c} l_{R}+\frac{y^{(2)}}{\Lambda} \Phi^{i} \bar{L}_{i}^{c} \gamma_{\mu} D^{\mu} l_{R}+\frac{1}{2} y^{(3)} \boldsymbol{\Phi}_{j}^{i} \bar{L}_{i}^{c} L^{j}+\text { h.c. }
$$

where the three quantities $y^{(1)}, y^{(2)}$ and $y^{(3)}$ are $3 \times 3$ matrices in generation space and where flavor indices have been omitted for clarity. Moreover, we have explicitly indicated the chirality of the lepton fields, or equivalently their $S U(2)_{L}$ representations so that there is no confusion about the action of the gauge-covariant derivative $D_{\mu}$. The (four-component) spinorial field $l_{R}$ stands hence for the right-handed charged lepton singlet of $S U(2)_{L}$ and the objects

$$
L^{i}=\left(\begin{array}{c}
\nu_{L} \\
l_{L}
\end{array}\right) \quad \text { and } \quad L_{i}=\varepsilon_{i j} L^{j}=\left(\begin{array}{c}
l_{L} \\
-\nu_{L}
\end{array}\right)
$$

are two representations of the weak doublet comprised of the left-handed neutrino $\nu_{L}$ and charged lepton $l_{L}$ fields. The terms included in the Lagrangian of Eq. (2.6) are consistent with gauge and Lorentz invariance, which leads to

\footnotetext{
${ }^{1}$ In our notations, we always employ Latin letters of the middle of the alphabet for fundamental indices and Latin letters of the beginning of the alphabet for adjoint indices.
} 
the appearance of the charge conjugation operator denoted by the superscript ${ }^{c}$. Moreover, care is taken so that each component of the new scalar fields is allowed to decay into Standard Model particles. In particular, this implies the use of a higher-dimensional operator suppressed by an effective scale $\Lambda$ for the coupling of the weak doublet $\Phi$ to the lepton fields since the four-component spinor product $\bar{\xi}_{R}^{c} \lambda_{L}=0$ for any fermionic fields.

The Lagrangian of Eq. (2.4) includes couplings of the new fields to the electroweak gauge bosons so that the former can then be produced at hadron colliders either from quark-antiquark scattering or through vector boson fusion. The only processes giving rise to a signature with three charged leptons or more consist of the pair production of two doubly-charged fields or of the associated production of one singly-charged and one doubly-charged state. Considering first the neutral current channels, $q \bar{q} \rightarrow \phi^{++} \phi^{--}, q \bar{q} \rightarrow \Phi^{++} \Phi^{--}$and $q \bar{q} \rightarrow \boldsymbol{\Phi}^{++} \boldsymbol{\Phi}^{--}$, the relevant partonic cross sections read, as a function of the partonic center-of-mass energy $\hat{s}$,

$$
\begin{aligned}
& \hat{\sigma}_{1}^{N C}=\frac{4 \pi \alpha^{2} \hat{s}}{9}\left[1-4 x_{\phi^{++}}^{2}\right]^{\frac{3}{2}}\left[\frac{e_{q}^{2}}{\hat{s}^{2}}-\frac{e_{q}\left(L_{q}+R_{q}\right)\left(\hat{s}-M_{Z}^{2}\right)}{2 c_{W}^{2} \hat{s}\left|\hat{s}_{Z}\right|^{2}}+\frac{L_{q}^{2}+R_{q}^{2}}{8 c_{W}^{4}\left|\hat{s}_{Z}\right|^{2}}\right], \\
& \hat{\sigma}_{2}^{N C}=\frac{4 \pi \alpha^{2} \hat{s}}{9}\left[1-4 x_{\Phi^{++}}^{2}\right]^{\frac{3}{2}}\left[\frac{e_{q}^{2}}{\hat{s}^{2}}+\frac{e_{q}\left(1-4 s_{W}^{2}\right)\left(L_{q}+R_{q}\right)\left(\hat{s}-M_{Z}^{2}\right)}{8 c_{W}^{2} s_{W}^{2} \hat{s}\left|\hat{s}_{Z}\right|^{2}}+\frac{\left(1-4 s_{W}^{2}\right)^{2}\left(L_{q}^{2}+R_{q}^{2}\right)}{128 c_{W}^{4} s_{W}^{4}\left|\hat{s}_{Z}\right|^{2}}\right], \\
& \hat{\sigma}_{3}^{N C}=\frac{4 \pi \alpha^{2} \hat{s}}{9}\left[1-4 x_{\Phi^{++}}^{2}\right]^{\frac{3}{2}}\left[\frac{e_{q}^{2}}{\hat{s}^{2}}+\frac{e_{q}\left(1-2 s_{W}^{2}\right)\left(L_{q}+R_{q}\right)\left(\hat{s}-M_{Z}^{2}\right)}{4 c_{W}^{2} s_{W}^{2} \hat{s}\left|\hat{s}_{Z}\right|^{2}}+\frac{\left(1-2 s_{W}^{2}\right)^{2}\left(L_{q}^{2}+R_{q}^{2}\right)}{32 c_{W}^{4} s_{W}^{4}\left|\hat{s}_{Z}\right|^{2}}\right],
\end{aligned}
$$

where $\hat{\sigma}_{i}$ is associated with the pair production of doubly-charged components of multiplets in the $\underset{\sim}{\mathbf{i}}$ representation of $S U(2)_{L}$. In those expressions, we have introduced the sine and cosine of the weak mixing angle $s_{W}$ and $c_{W}$, the quark electric charges $e_{q}$, their weak isospin quantum numbers $T_{3 q}$ and their $Z$-boson coupling strengths $L_{q}=2\left(T_{3 q}-e_{q} s_{W}^{2}\right)$ and $R_{q}=-2 e_{q} s_{W}^{2}$. Moreover, we are employing the reduced kinematical variables $x_{\phi}^{2}=\frac{M_{\phi}^{2}}{\hat{s}}$ and $\hat{s}_{Z}=\hat{s}_{-}-M_{Z}^{2}+i \Gamma_{Z} M_{Z}$, where $M_{Z}$ and $\Gamma_{Z}$ are the $Z$-boson mass and width, respectively.

For a new $S U(2)_{L}$ doublet (triplet) of scalar fields, trilepton signatures can also rise from the charged current production of a doubly-charged state in association with a singly-charged state, $u_{i} \bar{d}_{j} \rightarrow \Phi^{++} \Phi^{-}\left(u_{i} \bar{d}_{j} \rightarrow \boldsymbol{\Phi}^{++} \boldsymbol{\Phi}^{-}\right)$. The corresponding cross sections read

$$
\begin{aligned}
& \hat{\sigma}_{2}^{C C}=\frac{\pi \alpha^{2} \hat{s}}{72 s_{W}^{4}\left|\hat{s}_{W}\right|^{2}}\left|V_{i j}^{\mathrm{CKM}}\right|^{2} \lambda^{\frac{3}{2}}\left(1, x_{\Phi^{++}}^{2}, x_{\Phi^{+}}^{2}\right), \\
& \hat{\sigma}_{3}^{C C}=\frac{\pi \alpha^{2} \hat{s}}{36 s_{W}^{4}\left|\hat{s}_{W}\right|^{2}}\left|V_{i j}^{\mathrm{CKM}}\right|^{2} \lambda^{\frac{3}{2}}\left(1, x_{\Phi^{++}}^{2}, x_{\Phi^{+}}^{2}\right),
\end{aligned}
$$

where as for the neutral currents, we have employed the reduced propagator $\hat{s}_{W}=\hat{s}-M_{W}^{2}+i \Gamma_{W} M_{W}, M_{W}$ and $\Gamma_{W}$ being the $W$-boson mass and width, in addition to the Källen function $\lambda(a, b, c)=a^{2}+b^{2}+c^{2}-2 a b-2 b c-2 c a$ and to the CKM matrix $V^{\mathrm{CKM}}$.

After electroweak symmetry breaking, the neutral component of the $\boldsymbol{\Phi}$ multiplet can develop a vacuum expectation value (vev) $v_{\boldsymbol{\Phi}}$

$$
\boldsymbol{\Phi}^{0} \rightarrow \frac{1}{\sqrt{2}}\left[v_{\boldsymbol{\Phi}}+\mathbf{H}^{0}+i \mathbf{A}^{0}\right],
$$

where we are now distinguishing the scalar $\mathbf{H}^{0}$ and pseudoscalar $\mathbf{A}^{0}$ degrees of freedom of the neutral field. In principle, $v_{\boldsymbol{\Phi}}$ is constrained to be small by the electroweak $\rho$-parameter which only slightly deviates from unity 73 75, 81], in addition to strong constraints arising from the neutrino sector [17 20]. We have however decided to consider both scenarios with a very small $v_{\boldsymbol{\Phi}}$ value and those with a larger value, as in left-right symmetric models where the vacuum expectation values of the neutral fields are less constrained. In this case, the Lagrangian of Eq. (2.4) allows for the production of a single doubly-charged or singly-charged new state through vector boson fusion [27, 76] or in association with a weak gauge boson [77]. Since vector boson fusion processes do not yield final states with more than two charged leptons, we restrict ourselves to the study of the $q \bar{q}^{\prime} \rightarrow \boldsymbol{\Phi}^{+} Z$ and $q \bar{q}^{\prime} \rightarrow \boldsymbol{\Phi}^{++} W$ channels, the corresponding partonic cross sections being given by

$$
\begin{aligned}
\hat{\sigma}^{W \boldsymbol{\Phi}^{++}} & =\frac{\pi^{2} \alpha^{3} v_{\boldsymbol{\Phi}}^{2}}{18 s_{W}^{6} \hat{s}_{W}^{2}}\left|V_{i j}^{\mathrm{CKM}}\right|^{2} \lambda^{\frac{1}{2}}\left(1, x_{\Phi^{++}}^{2}, x_{W}^{2}\right)\left[\frac{\lambda\left(1, x_{\Phi^{++}}^{2}, x_{W}^{2}\right)}{x_{W}^{2}}+12\right], \\
\hat{\sigma}^{Z \boldsymbol{\Phi}^{+}} & =\frac{\pi^{2} \alpha^{3} v_{\boldsymbol{\Phi}}^{2}\left(1+s_{W}^{2}\right)^{2}}{72 s_{W}^{6} c_{W}^{2} \hat{s}_{W}^{2}}\left|V_{i j}^{\mathrm{CKM}}\right|^{2} \lambda^{\frac{1}{2}}\left(1, x_{\Phi^{++}}^{2}, x_{Z}^{2}\right)\left[\frac{\lambda\left(1, x_{\Phi^{++}}^{2}, x_{Z}^{2}\right)}{x_{Z}^{2}}+12\right] .
\end{aligned}
$$

In addition, the neutral $\mathbf{A}^{\mathbf{0}}$ and $\mathbf{H}^{\mathbf{0}}$ fields can also decay into multileptonic final states so that their single- and pair-production should be considered. However, a correct treatment of these fields implies to also add their mixings 
with the components of the Standard Model Higgs doublet. This renders the approach rather non-minimal so that we neglect them from the present analysis. Let us note that including them would not change our conclusions in the following sections and even increase any possible new physics signals.

As mentioned above, the Lagrangian of Eq. (2.6) allows for the new states to decay. We assume that these states are all close enough in mass so that they cannot decay into each other and compute below the relevant partial widths. Considering the decays of the doubly-charged states into a same-sign lepton pair, we obtain, assuming lepton flavor conservation,

$$
\begin{aligned}
& \Gamma_{1, \ell}^{++}=\frac{M_{\phi^{+}}\left|y^{(1)}\right|^{2}}{32 \pi}\left[1-2 x_{\ell}^{2}\right] \sqrt{1-4 x_{\ell}^{2}}, \\
& \Gamma_{2, \ell}^{++}=\frac{M_{\Phi^{++}} M_{\ell}^{2}\left|y^{(2)}\right|^{2}}{8 \pi \Lambda^{2}}\left[1-2 x_{\ell}^{2}\right] \sqrt{1-4 x_{\ell}^{2}}, \\
& \Gamma_{3, \ell}^{++}=\frac{M_{\Phi^{++}}\left|y^{(3)}\right|^{2}}{32 \pi}\left[1-2 x_{\ell}^{2}\right] \sqrt{1-4 x_{\ell}^{2}},
\end{aligned}
$$

using again different subscripts to distinguish the scalar field representations. Whereas flavor-violating effects could have also been considered, we nevertheless neglect them as they are constrained to be small due to lepton decay processes such as $\mu \rightarrow 3 e, \mu \rightarrow e \gamma$, etc., which are bound to be extremely rare [79 83]. In addition, the triplet field can also decay into a pair of $W^{+}$-bosons, the associated width being given by

$$
\Gamma_{3, W W}^{++}=\frac{M_{\mathbf{\Phi}++}^{3} \alpha^{2} \pi v_{\mathbf{\Phi}}^{2}}{4 M_{W}^{4} s_{W}^{4}} \sqrt{1-4 x_{W}^{2}}\left[1-4 x_{W}^{2}+12 x_{W}^{4}\right]
$$

Turning to the singly charged components of the new multiplets, the partial widths for the leptonic decays $\Phi^{+} \rightarrow \ell^{+} \bar{\nu}_{l}$ and $\boldsymbol{\Phi}^{+} \rightarrow \ell^{+} \bar{\nu}_{l}$ are computed as

$$
\begin{aligned}
& \Gamma_{2, \ell}^{+}=\frac{M_{\Phi^{+}} M_{\ell}^{2}\left|y^{(2)}\right|^{2}}{16 \pi \Lambda^{2}}\left[1-x_{\ell}^{2}\right]^{2}, \\
& \Gamma_{3, \ell}^{+}=\frac{M_{\Phi^{+}}\left|y^{(3)}\right|^{2}}{32 \pi}\left[1-x_{\ell}^{2}\right]^{2},
\end{aligned}
$$

while the one for the decay of a triplet field into a pair of weak gauge bosons, $\boldsymbol{\Phi}^{+} \rightarrow W^{+} Z$, is found to be

$$
\Gamma_{3, W Z}^{+}=\frac{M_{\mathbf{\Phi}^{+}}^{3} \alpha^{2} \pi v_{\mathbf{\Phi}}^{2}\left(1+s_{W}^{2}\right)^{2}}{8 c_{W}^{2} s_{W}^{4} M_{Z}^{2} M_{W}^{2}}\left[\lambda\left(1, x_{W}^{2}, x_{Z}^{2}\right)+12 x_{Z}^{2} x_{W}^{2}\right] \sqrt{\lambda\left(1, x_{W}^{2}, x_{Z}^{2}\right)} .
$$

\subsection{Spin $1 / 2$ doubly-charged particles}

\subsubsection{Simplified models with three generations of leptons}

We now turn to the building of Standard Model extensions containing one extra fermionic multiplet and like in Section 2.1. we restrict ourselves to states with an electric charge not higher than two. We therefore consider three fermionic fields $\psi, \Psi$ and $\boldsymbol{\Psi}$ lying in the singlet, doublet and adjoint representation of $S U(2)_{L}$, respectively, and we fix their hypercharge quantum numbers to $Y_{\psi}=2, Y_{\Psi}=3 / 2$ and $Y_{\Psi}=1$,

$$
\psi \equiv \psi^{++}, \quad \Psi^{i} \equiv\left(\begin{array}{c}
\Psi^{++} \\
\Psi^{+}
\end{array}\right) \quad \text { and } \quad \Psi_{j}^{i} \equiv\left(\begin{array}{cc}
\frac{\Psi^{+}}{\sqrt{2}} & \boldsymbol{\Psi}^{++} \\
\boldsymbol{\Psi}^{0} & -\frac{\boldsymbol{\Psi}^{+}}{\sqrt{2}}
\end{array}\right)
$$

The associated kinetic and gauge interaction terms are standard and induced by gauge covariant derivatives that can be derived from Eq. (2.5),

$$
\mathcal{L}_{\text {kin }}=i \bar{\psi} \gamma^{\mu} D_{\mu} \psi+i \bar{\Psi}_{i} \gamma^{\mu} D_{\mu} \Psi^{i}+i \bar{\Psi}_{a} \gamma^{\mu} D_{\mu} \Psi^{a}+\ldots
$$

where mass terms are included in the dots. As in Section 2.1, we assume the new states to be almost mass-degenerate so that they cannot decay into each other. In order to allow for the decays of the $\psi, \Psi$ and $\boldsymbol{\Psi}$ states, it is thus necessary to introduce at least one additional fermionic particle $N$, which we choose to be gauge singlet as in massive neutrino models 84 86]. Avoiding the introduction of more new states to our simplified model, three-body decays of the $\psi$, 
$\Psi$ and $\Psi$ fermions into a lepton pair and a $N$ particle are permitted by means of non-renormalizable four-fermion interactions,

$$
\begin{aligned}
\mathcal{L}_{\mathrm{F}} & =\frac{G^{(1,1)}}{2 \Lambda^{2}}\left[\bar{l}_{R}^{c} l_{R}\right]\left[\bar{N} P_{L} \psi\right]+\frac{G^{(1,2)}}{2 \Lambda^{2}}\left[\bar{l}_{R}^{c} l_{R}\right]\left[\bar{N} P_{R} \psi\right]+\frac{G^{(2,1)}}{\Lambda^{2}}\left[\bar{l}_{R}^{c} \Psi^{i}\right]\left[\bar{N} L_{i}\right]+\frac{G^{(2,2)}}{\Lambda^{2}}\left[\bar{l}_{R}^{c} N\right]\left[\bar{L}^{i c} \Psi_{i}\right] \\
& +\frac{G^{(3,1)}}{2 \Lambda^{2}}\left[\bar{L}_{i}^{c} L^{j}\right]\left[\bar{N} P_{L} \Psi^{i}{ }_{j}\right]+\frac{G^{(3,2)}}{2 \Lambda^{2}}\left[\bar{L}_{i}^{c} L^{j}\right]\left[\bar{N} P_{R} \Psi^{i}{ }_{j}\right]+\text { h.c. } .
\end{aligned}
$$

In this Lagrangian, we have only considered a set of independent effective operators and omitted all generation indices for clarity. In addition, $P_{L}$ and $P_{R}$ are chirality projectors acting on spin space, the left-handed and right-handed fermionic components of the Standard Model fields have been defined in Section 2.1 and the interaction strengths suppressed by a new physics scale $\Lambda$ are $3 \times 3$ matrices in flavor space $G^{(1,1)}, G^{(1,2)}, G^{(2,1)}, G^{(2,2)}, G^{(3,1)}$ and $G^{(3,2)}$.

The gauge interactions included in the Lagrangian of Eq. (2.17) imply the possible hadroproduction of pairs of $\psi$, $\Psi$ and $\boldsymbol{\Psi}$ states from quark-antiquark scattering. Focusing on final state signatures with at least three leptons, the partonic cross sections associated with the relevant neutral current processes $q \bar{q} \rightarrow \psi^{++} \psi^{--}, q \bar{q} \rightarrow \Psi^{++} \Psi^{--}$and $q \bar{q} \rightarrow \boldsymbol{\Psi}^{++} \boldsymbol{\Psi}^{--}$are

$$
\begin{aligned}
& \hat{\sigma}_{1}^{N C}=\frac{16 \pi \alpha^{2} \hat{s}}{9}\left[1+2 x_{\psi^{++}}^{2}\right] \sqrt{1-4 x_{\psi^{++}}^{2}}\left[\frac{e_{q}^{2}}{\hat{s}^{2}}-\frac{e_{q}\left(L_{q}+R_{q}\right)\left(\hat{s}-M_{Z}^{2}\right)}{2 c_{W}^{2} \hat{s}\left|\hat{s}_{Z}\right|^{2}}+\frac{L_{q}^{2}+R_{q}^{2}}{8 c_{W}^{4}\left|\hat{s}_{Z}\right|^{2}}\right], \\
& \hat{\sigma}_{2}^{N C}=\frac{16 \pi \alpha^{2} \hat{s}}{9}\left[1+2 x_{\Psi^{++}}^{2}\right] \sqrt{1-4 x_{\Psi^{++}}^{2}}\left[\frac{e_{q}^{2}}{\hat{s}^{2}}+\frac{e_{q}\left(1-4 s_{W}^{2}\right)\left(L_{q}+R_{q}\right)\left(\hat{s}-M_{Z}^{2}\right)}{8 c_{W}^{2} s_{W}^{2} \hat{s}\left|\hat{s}_{Z}\right|^{2}}+\frac{\left(1-4 s_{W}^{2}\right)^{2}\left(L_{q}^{2}+R_{q}^{2}\right)}{128 c_{W}^{4} s_{W}^{4}\left|\hat{s}_{Z}\right|^{2}}\right], \\
& \hat{\sigma}_{3}^{N C}=\frac{16 \pi \alpha^{2} \hat{s}}{9}\left[1+2 x_{\Psi^{++}}^{2}\right] \sqrt{1-4 x_{\Psi^{++}}^{2}}\left[\frac{e_{q}^{2}}{\hat{s}^{2}}+\frac{e_{q}\left(1-2 s_{W}^{2}\right)\left(L_{q}+R_{q}\right)\left(\hat{s}-M_{Z}^{2}\right)}{\left.4 c_{W}^{2} s_{W}^{2} \hat{s}^{\mid} \hat{s}_{Z}\right|^{2}}+\frac{\left(1-2 s_{W}^{2}\right)^{2}\left(L_{q}^{2}+R_{q}^{2}\right)}{32 c_{W}^{4} s_{W}^{4}\left|\hat{s}_{Z}\right|^{2}}\right],
\end{aligned}
$$

whereas those related to the charged current processes $u_{i} \bar{d}_{j} \rightarrow \Psi^{++} \Psi^{-}$and $u_{i} \bar{d}_{j} \rightarrow \boldsymbol{\Psi}^{++} \boldsymbol{\Psi}^{-}$are

$$
\begin{aligned}
& \hat{\sigma}_{2}^{C C}=\frac{\pi \alpha^{2} \hat{s}}{36 s_{W}^{4}\left|\hat{s}_{W}\right|^{2}}\left|V_{i j}^{C K M}\right|^{2} \sqrt{\lambda\left(1, x_{\Psi^{++}}^{2}, x_{\Psi^{+}}^{2}\right)}\left[1-\left(x_{\Psi^{++}}-x_{\Psi^{+}}\right)^{2}\right]\left[2+\left(x_{\Psi^{++}}+x_{\Psi^{+}}\right)^{2}\right], \\
& \hat{\sigma}_{3}^{C C}=\frac{\pi \alpha^{2} \hat{s}}{18 s_{W}^{4}\left|\hat{s}_{W}\right|^{2}}\left|V_{i j}^{\mathrm{CKM}}\right|^{2} \sqrt{\lambda\left(1, x_{\Psi^{++}}^{2}, x_{\Psi^{+}}^{2}\right)}\left[1-\left(x_{\Psi^{++}}-x_{\Psi^{+}}\right)^{2}\right]\left[2+\left(x_{\Psi^{++}}+x_{\Psi^{+}}\right)^{2}\right] .
\end{aligned}
$$

We recall that our conventions for the notations have been introduced in Section 2.1. Since there are no closed formulas for the widths of the new particles that can be calculated from Eq. (2.18), we refer to Section 3 for the corresponding numerical analysis. As in the scalar case, flavor violating effects are again neglected since they are constrained from rare leptonic decays, as illustrated in the left-right supersymmetric case in Ref. [87].

\subsubsection{Simplified models with four charged lepton species}

Since the singly-charged component of the $\Psi$ and $\Psi$ multiplets have the same quantum numbers as the charged leptons, they could mix, as in $R$-parity violating supersymmetric theories [78] or in custodian tau models [36]. This mixing is however highly constrained by LEP data, by measurements of the muon anomalous magnetic moment as well as by limits on leptonic flavor violating processes and on conversions in nuclei. We therefore restrict the most general case to a $2 \times 2$ mixing with tau leptons,

$$
\left(\begin{array}{c}
e^{\prime} \\
\mu^{\prime} \\
\tau^{\prime} \\
E^{\prime}
\end{array}\right)=\left(\begin{array}{c}
e \\
\mu \\
\tau^{\prime} \\
E^{\prime}
\end{array}\right)=\left(\begin{array}{cccc}
1 & 0 & 0 & 0 \\
0 & 1 & 0 & 0 \\
0 & 0 & c_{\tau} & s_{\tau} \\
0 & 0 & -s_{\tau} & c_{\tau}
\end{array}\right)\left(\begin{array}{c}
e \\
\mu \\
\tau \\
\Psi^{+}
\end{array}\right) \quad \text { and } \quad\left(\begin{array}{c}
e^{\prime \prime} \\
\mu^{\prime \prime} \\
\tau^{\prime \prime} \\
E^{\prime \prime}
\end{array}\right)=\left(\begin{array}{c}
e \\
\mu \\
\tau^{\prime \prime} \\
E^{\prime \prime}
\end{array}\right)=\left(\begin{array}{cccc}
1 & 0 & 0 & 0 \\
0 & 1 & 0 & 0 \\
0 & 0 & c_{\tau} & s_{\tau} \\
0 & 0 & -s_{\tau} & c_{\tau}
\end{array}\right)\left(\begin{array}{c}
e \\
\mu \\
\tau \\
\Psi^{+}
\end{array}\right)
$$

where $c_{\tau}\left(s_{\tau}\right)$ denotes the cosine (sine) of the associated mixing angle. In those notations, the $E^{\prime}$ and $\tau^{\prime}$ fields are related to lepton mixing with the singly-charged component of the $S U(2)_{L}$ doublet $\Psi$ while the $E^{\prime \prime}$ and $\tau^{\prime \prime}$ fields to mixing with the $S U(2)_{L}$ triplet $\boldsymbol{\Psi}$. In contrast to the model constructed in Section 2.2.1 the decays of the new fields are possible via this mixing, without requiring the addition of any extra particle to the theory.

Assuming mass-degenerate $\Psi$ states, the doubly-charged component of the $S U(2)_{L}$ doublet always decays into a $W$-boson and a $\tau$ lepton, which further yields a final state with zero, one or two charged leptons. The associated partial width can be computed from the Lagrangian of Eq. (2.17) and is

$$
\Gamma_{2, \tau W}^{++}=\frac{M_{\Psi^{++}}^{3} \alpha s_{\tau}^{2}}{8 M_{W}^{2} s_{w}^{2}} \sqrt{\lambda\left(1, x_{W}^{2}, x_{\tau}^{2}\right)}\left[\lambda\left(1, x_{W}^{2}, x_{\tau}^{2}\right)-3 x_{W}^{2}\left(x_{W}^{2}-\left(1-x_{\tau}\right)^{2}\right)\right] .
$$


Similarly, the $E^{\prime}$ lepton can decay either to a $\tau Z$ or to a $\nu_{\tau} W$ final state, the corresponding widths being

$$
\begin{aligned}
\Gamma_{2, \tau Z}^{+} & =\frac{M_{E^{\prime}}^{3} \alpha s_{\tau}^{2} c_{\tau}^{2}}{32 M_{Z}^{2} c_{W}^{2} s_{W}^{2}} \sqrt{\lambda\left(1, x_{Z}^{2}, x_{\tau}^{2}\right)}\left[5 \lambda\left(1, x_{Z}^{2}, x_{\tau}^{2}\right)+3 x_{Z}^{2}\left(5\left(1+x_{\tau}^{2}-x_{Z}^{2}\right)-8 x_{\tau}\right)\right], \\
\Gamma_{2, \nu_{\tau} W}^{+} & =\frac{M_{E^{\prime}}^{3} \alpha s_{\tau}^{2}}{16 M_{W}^{2} s_{W}^{2}}\left[1-x_{W}^{2}\right]^{2}\left[1+2 x_{W}^{2}\right] .
\end{aligned}
$$

After accounting for the decays of the Standard Model particles, other production mechanisms, in addition to the one of a pair of doubly charged states (whose cross section is given by Eq. (2.19)), can induce signatures with at least three leptons. The two processes $q \bar{q} \rightarrow E^{\prime+} E^{\prime-}$ and $q \bar{q} \rightarrow E^{\prime \pm} \tau^{\mp}$ hence possibly lead to final states containing up to six leptons, the corresponding partonic cross sections being respectively given by

$$
\begin{aligned}
\hat{\sigma}_{2}^{E^{\prime} E^{\prime}}= & \frac{4 \pi \alpha^{2} \hat{s}}{9} \sqrt{1-4 x_{E^{\prime}}^{2}}\left[\left[1+2 x_{E^{\prime}}^{2}\right] \frac{e_{q}^{2}}{\hat{s}^{2}}-\frac{e_{q}\left(L_{q}+R_{q}\right)}{8 c_{W}^{2} s_{W}^{2}} \frac{\hat{s}-M_{Z}^{2}}{\hat{s}_{\left|\hat{s}_{Z}\right|^{2}}^{2}}\left[1+2 x_{E^{\prime}}^{2}\right]\left[2+4 s_{W}^{2}-3 s_{\tau}^{2}\right]\right. \\
& \left.+\frac{\left(L_{q}^{2}+R_{q}^{2}\right)}{64 c_{W}^{4} s_{W}^{4}} \frac{1}{\left|\hat{s}_{Z}\right|^{2}}\left(\left[1+2 x_{E^{\prime}}^{2}\right]\left[2\left(1+2 s_{W}^{2}\right)^{2}-6 s_{\tau}^{2}\left(1+2 s_{W}^{2}\right)\right]+s_{\tau}^{4}\left(5+7 x_{E^{\prime}}^{2}\right)\right)\right] \\
\hat{\sigma}_{2}^{E^{\prime} \tau}= & \frac{\pi \alpha^{2}\left(L_{q}^{2}+R_{q}^{2}\right) s_{\tau}^{2} c_{\tau}^{2}}{288 c_{W}^{4} s_{W}^{4}} \frac{\hat{s}}{\left|\hat{s}_{Z}\right|^{2}} \sqrt{\lambda\left(1, x_{\tau}^{2}, x_{E^{\prime}}^{2}\right)}\left[5\left(3-3 x_{E^{\prime}}^{2}-3 x_{\tau}^{2}-\lambda\left(1, x_{\tau}^{2}, x_{E^{\prime}}^{2}\right)\right)+24 x_{E^{\prime}} x_{\tau}\right]
\end{aligned}
$$

whilst the production channels of an associated pair comprised of a singly and a doubly-charged particle, $u_{i} \bar{d}_{j} \rightarrow$ $\Psi^{++} E^{\prime-}$ and $u_{i} \bar{d}_{j} \rightarrow \Psi^{++} \tau^{-}$, can yield up to five charged leptons. In this case, the production cross sections are

$$
\begin{aligned}
\hat{\sigma}_{2}^{\Psi^{++} E^{\prime}} & =\frac{\pi \alpha^{2} \hat{s} c_{\tau}^{2}}{36 s_{W}^{4}\left|\hat{s}_{W}\right|^{2}}\left|V_{i j}^{\mathrm{CKM}}\right|^{2} \sqrt{\lambda\left(1, x_{\Psi^{++}}^{2}, x_{E^{\prime}}^{2}\right)}\left[1-\left(x_{\Psi^{++}}-x_{E^{\prime}}\right)^{2}\right]\left[2+\left(x_{\Psi^{++}}+x_{E^{\prime}}\right)^{2}\right], \\
\hat{\sigma}_{2}^{\Psi^{++}} \tau & =\frac{\pi \alpha^{2} \hat{s} s_{\tau}^{2}}{36 s_{W}^{4}\left|\hat{s}_{W}\right|^{2}}\left|V_{i j}^{\mathrm{CKM}}\right|^{2} \sqrt{\lambda\left(1, x_{\Psi^{++}}^{2}, x_{\tau}^{2}\right)}\left[1-\left(x_{\Psi^{++}}-x_{\tau}\right)^{2}\right]\left[2+\left(x_{\Psi^{++}}+x_{\tau}\right)^{2}\right] .
\end{aligned}
$$

In the same way, all the components of the $\boldsymbol{\Psi}$ multiplet can decay to final states containing up to three leptons, after accounting for subsequent decays of the gauge bosons and tau leptons. The corresponding partial widths are

$$
\begin{aligned}
\Gamma_{3, \tau W}^{++} & =\frac{M_{\psi^{++}}^{3} \alpha s_{\tau}^{2}}{4 M_{W}^{2} s_{W}^{2}} \sqrt{\lambda\left(1, x_{W}^{2}, x_{\tau}^{2}\right)}\left[\lambda\left(1, x_{W}^{2}, x_{\tau}^{2}\right)+3 x_{W}^{2}\left(\left(1-x_{\tau}\right)^{2}-x_{W}^{2}\right)\right], \\
\Gamma_{3, \tau Z}^{+} & =\frac{M_{E^{\prime \prime}}^{3} \alpha s_{\tau}^{2} c_{\tau}^{2}}{32 M_{Z}^{2} c_{W}^{2} s_{W}^{2}} \sqrt{\lambda\left(1, x_{Z}^{2}, x_{\tau}^{2}\right)}\left[\lambda\left(1, x_{Z}^{2}, x_{\tau}^{2}\right)+3 x_{Z}^{2}\left(1+x_{\tau}^{2}-x_{Z}^{2}\right)\right], \\
\Gamma_{3, \nu_{\tau} W}^{+} & =\frac{M_{E^{\prime \prime}}^{3} \alpha s_{\tau}^{2}}{16 M_{W}^{2} s_{W}^{2}}\left[1-x_{W}^{2}\right]^{2}\left[1+2 x_{W}^{2}\right], \\
\Gamma_{3, \tau W}^{0} & =\frac{M_{\psi^{0}}^{3} \alpha s_{\tau}^{2}}{4 M_{W}^{2} s_{W}^{2}} \sqrt{\lambda\left(1, x_{W}^{2}, x_{\tau}^{2}\right)}\left[\lambda\left(1, x_{W}^{2}, x_{\tau}^{2}\right)+3 x_{W}^{2}\left(\left(1-x_{\tau}\right)^{2}-x_{W}^{2}\right)\right] .
\end{aligned}
$$

Therefore, the production of any pair of components of the $\boldsymbol{\Psi}$ field can lead to signatures with three or more charged leptons. While the partonic cross section related to the production of two doubly-charged particles is given in Eq. (2.19), all the other relevant cross sections, associated with the $u_{i} \bar{d}_{j} \rightarrow \boldsymbol{\Psi}^{++} E^{\prime \prime-}, u_{i} \bar{d}_{j} \rightarrow \boldsymbol{\Psi}^{++} \tau^{-}, q \bar{q} \rightarrow E^{\prime \prime+} E^{\prime \prime-}$, 
$q \bar{q} \rightarrow E^{\prime \prime \pm} \tau^{\mp}, u_{i} \bar{d}_{j} \rightarrow \boldsymbol{\Psi}^{0} E^{\prime \prime+}, u_{i} \bar{d}_{j} \rightarrow \boldsymbol{\Psi}^{0} \tau^{+}$and $q \bar{q} \rightarrow \boldsymbol{\Psi}^{0} \boldsymbol{\Psi}^{0}$ modes, are respectively computed as

$$
\begin{aligned}
\hat{\sigma}_{3}^{\Psi^{++} E^{\prime \prime}=} & \frac{\pi \alpha^{2} \hat{s} c_{\tau}^{2}}{18 s_{W}^{4}\left|\hat{s}_{W}\right|^{2}}\left|V_{i j}^{\mathrm{CKM}}\right|^{2} \sqrt{\lambda\left(1, x_{\Psi^{++}}^{2}, x_{E^{\prime \prime}}^{2}\right)}\left[1-\left(x_{\Psi^{++}}-x_{E^{\prime \prime}}\right)^{2}\right]\left[2+\left(x_{\Psi^{++}}+x_{E^{\prime \prime}}\right)^{2}\right], \\
\hat{\sigma}_{3}^{\Psi^{++} \tau}= & \frac{\pi \alpha^{2} \hat{s} s_{\tau}^{2}}{18 s_{W}^{4}\left|\hat{s}_{W}\right|^{2}}\left|V_{i j}^{\mathrm{CKM}}\right|^{2} \sqrt{\lambda\left(1, x_{\Psi^{++}}^{2}, x_{\tau}^{2}\right)}\left[1-\left(x_{\Psi^{++}}-x_{\tau}\right)^{2}\right]\left[2+\left(x_{\Psi^{++}}+x_{\tau}\right)^{2}\right], \\
\hat{\sigma}_{3}^{E^{\prime \prime} E^{\prime \prime}=}= & \frac{4 \pi \alpha^{2} \hat{s}}{9} \sqrt{1-4 x_{E^{\prime \prime}}^{2}}\left[\left[1+2 x_{E^{\prime \prime}}^{2}\right] \frac{e_{q}^{2}}{\hat{s}^{2}}-\frac{e_{q}\left(L_{q}+R_{q}\right)}{8 c_{W}^{2} s_{W}^{2}} \frac{\hat{s}-M_{Z}^{2}}{\hat{s}_{\mid}\left|\hat{s}_{Z}\right|^{2}}\left[1+2 x_{E^{\prime \prime}}^{2}\right]\left[4 s_{W}^{2}-s_{\tau}^{2}\right]\right. \\
& \left.+\frac{\left(L_{q}^{2}+R_{q}^{2}\right)}{64 c_{W}^{4} s_{W}^{4}} \frac{1}{\left|\hat{s}_{Z}\right|^{2}}\left(\left[1+2 x_{E^{\prime \prime}}^{2}\right]\left[8 s_{W}^{4}-4 s_{\tau}^{2} s_{W}^{2}\right]+s_{\tau}^{4}\left(1-x_{E^{\prime \prime}}^{2}\right)\right)\right], \\
\hat{\sigma}_{3}^{E^{\prime \prime} \tau}= & \frac{\pi \alpha^{2}\left(L_{q}^{2}+R_{q}^{2}\right) s_{\tau}^{2} c_{\tau}^{2}}{288 c_{W}^{4} s_{W}^{4}} \frac{\hat{s}}{\left|\hat{s}_{Z}\right|^{2}} \sqrt{\lambda\left(1, x_{\tau}^{2}, x_{E^{\prime \prime}}^{2}\right)}\left[3\left(1-x_{E^{\prime \prime}}^{2}-x_{\tau}^{2}\right)-\lambda\left(1, x_{\tau}^{2}, x_{E^{\prime \prime}}^{2}\right)\right], \\
\hat{\sigma}_{3}^{E^{\prime \prime} \Psi^{0}}= & \frac{\pi \alpha^{2} \hat{s}_{\tau}^{2}}{18 s_{W}^{4}\left|\hat{s}_{W}\right|^{2}}\left|V_{i j}^{\mathrm{CKM}}\right|^{2} \sqrt{\lambda\left(1, x_{\Psi^{0}}^{2}, x_{E^{\prime \prime}}^{2}\right)}\left[1-\left(x_{\Psi^{0}}-x_{E^{\prime \prime}}\right)^{2}\right]\left[2+\left(x_{\Psi^{0}}+x_{E^{\prime \prime}}\right)^{2}\right], \\
\hat{\sigma}_{3}^{\tau \Psi^{0}}= & \frac{\pi \alpha^{2} \hat{s} s_{\tau}^{2}}{18 s_{W}^{4}\left|\hat{s}_{W}\right|^{2}}\left|V_{i j}^{\mathrm{CKM}}\right|^{2} \sqrt{\lambda\left(1, x_{\Psi^{0}}^{2}, x_{\tau}^{2}\right)}\left[1-\left(x_{\Psi^{0}}-x_{\tau}\right)^{2}\right]\left[2+\left(x_{\Psi^{0}}+x_{\tau}\right)^{2}\right], \\
\hat{\sigma}_{3}^{\Psi^{0} \Psi^{0}}= & \frac{\pi \alpha^{2}\left(L_{q}^{2}+R_{q}^{2}\right)}{18 c_{W}^{4} s_{W}^{4}} \frac{\hat{s}}{\left|\hat{s}_{Z}\right|^{2}} \sqrt{1-4 x_{\Psi^{0}}^{2}}\left[1+2 x_{\Psi^{0}}^{2}\right] .
\end{aligned}
$$

\subsection{Spin 1 doubly-charged particles}

In this section, we adjoin to the Standard Model particle content additional complex vectorial fields $V, \mathcal{V}$ and V lying respectively in the singlet, fundamental and adjoint representation of $S U(2)_{L}$. The hypercharge quantum numbers are again set to $Y_{V}=2, Y_{\mathcal{V}}=3 / 2$ and $Y_{\mathbf{V}}=1$, so that the component fields read

$$
V_{\mu} \equiv V_{\mu}^{++}, \quad \mathcal{V}_{\mu}^{i} \equiv\left(\begin{array}{c}
\mathcal{V}_{\mu}^{++} \\
\mathcal{V}_{\mu}^{+}
\end{array}\right) \quad \text { and } \quad \mathbf{V}^{i}{ }_{j} \equiv\left(\begin{array}{cc}
\frac{\mathbf{V}_{\mu}^{+}}{\sqrt{2}} & \mathbf{V}_{\mu}^{++} \\
\mathbf{V}_{\mu}^{0} & -\frac{\mathbf{V}_{\mu}^{+}}{\sqrt{2}}
\end{array}\right) .
$$

Gauge interactions and kinetic terms are described by means of a gauge-covariant version of the Proca Lagrangian

$$
\begin{aligned}
\mathcal{L}_{\text {kin }}= & -\frac{1}{2}\left[D_{\mu} V_{\nu}^{\dagger}-D_{\nu} V_{\mu}^{\dagger}\right]\left[D^{\mu} V^{\nu}-D^{\nu} V^{\mu}\right]-\frac{1}{2}\left[D_{\mu} \mathcal{V}_{\nu}^{\dagger}-D_{\nu} \mathcal{V}_{\mu}^{\dagger}\right]\left[D^{\mu} \mathcal{V}^{\nu}-D^{\nu} \mathcal{V}^{\mu}\right] \\
& -\frac{1}{2}\left[D_{\mu} \mathbf{V}_{\nu}^{\dagger}-D_{\nu} \mathbf{V}_{\mu}^{\dagger}\right]\left[D^{\mu} \mathbf{V}^{\nu}-D^{\nu} \mathbf{V}^{\mu}\right]+\ldots,
\end{aligned}
$$

the dots being identified with omitted mass terms and the covariant derivatives being derived from Eq. (2.5). As in

the previous sections, we again forbid the new states to decay into each other and model the decays of the new vector fields into pairs of charged leptons via the Lagrangian

$$
\mathcal{L}_{\mathrm{dec}}=\frac{\tilde{g}^{(1)}}{\Lambda} V_{\mu} \bar{l}_{R}^{c} \sigma^{\mu \nu} D_{\nu} l_{R}+\tilde{g}^{(2)} \mathcal{V}_{\mu}^{i} \bar{L}_{i}^{c} \gamma^{\mu} l_{R}+\frac{\tilde{g}^{(3)}}{\Lambda}\left(\mathbf{V}_{\mu}\right)^{i}{ }_{j} \bar{L}_{i}^{c} \sigma^{\mu \nu} D_{\nu} L^{j}
$$

where the coupling strengths are denoted by $3 \times 3$ matrices in flavor space $\tilde{g}^{(1)}, \tilde{g}^{(2)}$ and $\tilde{g}^{(3)}$ and where $\sigma^{\mu \nu}=\frac{i}{4}\left[\gamma^{\mu}, \gamma^{\nu}\right]$. Gauge invariance makes use of the standard gauge-covariant derivatives $D_{\mu} L$ and $D_{\mu} l_{R}$ as well as of the dual object $L_{i}$ defined in Eq. (2.7). In addition, dimension-four operators such as $\bar{l}_{R}^{c} \gamma^{\mu} l_{R}$ identically vanish so that the use of higher-dimensional operators suppressed by a new physics scale $\Lambda$ is required.

The Lagrangian of Eq. (2.28) allows to pair produce the new doubly charged fields via quark-antiquark scattering processes, $q \bar{q} \rightarrow V^{++} V^{--}, q \bar{q} \rightarrow \mathcal{V}^{++} \mathcal{V}^{--}$and $q \bar{q} \rightarrow \mathbf{V}^{++} \mathbf{V}^{--}$, which leads to a four leptons signature after accounting for the decays permitted by the interactions described in Eq. (2.30). The associated partonic cross sections 
are calculated as

$$
\begin{aligned}
\hat{\sigma}_{1}^{N C}= & \frac{4 \pi \alpha^{2} \hat{s}}{9} \sqrt{1-4 x_{V^{++}}^{2}} \frac{1-x_{V^{++}}^{2}-12 x_{V^{++}}^{4}}{x_{V^{++}}^{2}}\left[\frac{e_{q}^{2}}{\hat{s}^{2}}-\frac{e_{q}\left(L_{q}+R_{q}\right)\left(\hat{s}-M_{Z}^{2}\right)}{2 c_{W}^{2} \hat{s}^{\mid}\left|\hat{s}_{Z}\right|^{2}}+\frac{L_{q}^{2}+R_{q}^{2}}{8 c_{W}^{4}\left|\hat{s}_{Z}\right|^{2}}\right], \\
\hat{\sigma}_{2}^{N C}= & \frac{4 \pi \alpha^{2} \hat{s}}{9} \sqrt{1-4 x_{\mathcal{V}^{++}}^{2}} \frac{1-x_{\mathcal{V}^{++}}^{2}-12 x_{\mathcal{V}^{++}}^{4}}{x_{\mathcal{V}^{++}}^{2}} \\
& \times\left[\frac{e_{q}^{2}}{\hat{s}^{2}}+\frac{e_{q}\left(1-4 s_{W}^{2}\right)\left(L_{q}+R_{q}\right)\left(\hat{s}-M_{Z}^{2}\right)}{8 s_{W}^{2} c_{W}^{2} \hat{s}_{\mid}\left|\hat{s}_{Z}\right|^{2}}+\frac{\left(1-4 s_{W}^{2}\right)^{2}\left(L_{q}^{2}+R_{q}^{2}\right)}{128 s_{W}^{4} c_{W}^{4}\left|\hat{s}_{Z}\right|^{2}}\right], \\
\hat{\sigma}_{3}^{N C}= & \frac{4 \pi \alpha^{2} \hat{s}}{9} \sqrt{1-4 x_{\mathbf{V}^{++}}^{2}} \frac{1-x_{\mathbf{V}^{++}}^{2}-12 x_{\mathbf{V}^{++}}^{4}}{x_{\mathbf{V}^{++}}^{2}} \\
& \times\left[\frac{e_{q}^{2}}{\hat{s}^{2}}+\frac{e_{q}\left(1-2 s_{W}^{2}\right)\left(L_{q}+R_{q}\right)\left(\hat{s}-M_{Z}^{2}\right)}{4 s_{W}^{2} c_{W}^{2} \hat{s}^{2}\left|\hat{s}_{Z}\right|^{2}}+\frac{\left(1-2 s_{W}^{2}\right)^{2}\left(L_{q}^{2}+R_{q}^{2}\right)}{32 s_{W}^{4} c_{W}^{4}\left|\hat{s}_{Z}\right|^{2}}\right],
\end{aligned}
$$

respectively. In the case of fields lying in the fundamental or adjoint representation of $S U(2)_{L}$, three lepton final states also result from the production of an associated pair comprised of one doubly-charged state and one singly charged state, $u_{i} \bar{d}_{j} \rightarrow \mathcal{V}^{++} \mathcal{V}^{-}$and $u_{i} \bar{d}_{j} \rightarrow \mathbf{V}^{++} \mathbf{V}^{-}$whose cross sections respectively are

$$
\begin{aligned}
\hat{\sigma}_{2}^{C C}= & \frac{\pi \alpha^{2} \hat{s}}{288 s_{W}^{4}\left|\hat{s}_{W}\right|^{2}}\left|V_{i j}^{\mathrm{CKM}}\right|^{2} \sqrt{\lambda\left(1, x_{\mathcal{V}^{++}}^{2}, x_{\mathcal{V}^{+}}^{2}\right)} \frac{\left[1-\left(x_{\mathcal{V}^{++}}-x_{\mathcal{V}^{+}}\right)^{2}\right]\left[1-\left(x_{\mathcal{V}^{++}}+x_{\mathcal{V}^{+}}\right)^{2}\right]}{x_{\mathcal{V}^{++}}^{2} x_{\mathcal{V}^{+}}^{2}} \\
& \times\left[\lambda\left(1, x_{\mathcal{V}^{++}}^{2}, x_{\mathcal{V}^{+}}^{2}\right)-1+4\left(x_{\mathcal{V}^{+}}^{2}+x_{\mathcal{V}^{++}}^{2}+3 x_{\mathcal{V}^{+}}^{2} x_{\mathcal{V}^{++}}^{2}\right)\right] \\
\hat{\sigma}_{3}^{C C}= & \frac{\pi \alpha^{2} \hat{s}}{144 s_{W}^{4}\left|\hat{s}_{W}\right|^{2}}\left|V_{i j}^{C K M}\right|^{2} \sqrt{\lambda\left(1, x_{\mathbf{V}^{++}}^{2}, x_{\mathbf{V}^{+}}^{2}\right)} \frac{\left[1-\left(x_{\mathbf{V}^{++}}-x_{\mathbf{V}^{+}}\right)^{2}\right]\left[1-\left(x_{\mathbf{V}^{++}}+x_{\mathbf{V}^{+}}\right)^{2}\right]}{x_{\mathbf{V}^{++}}^{2} x_{\mathbf{V}^{+}}^{2}} \\
& \times\left[\lambda\left(1, x_{\mathbf{V}^{++}}^{2}, x_{\mathbf{V}^{+}}^{2}\right)-1+4\left(x_{\mathbf{V}^{+}}^{2}+x_{\mathbf{V}^{++}}^{2}+3 x_{\mathbf{V}^{+}}^{2} x_{\mathbf{V}^{++}}^{2}\right)\right] .
\end{aligned}
$$

The partial widths associated with the decays of the states above into charged leptons are finally given by

$$
\begin{aligned}
\Gamma_{1, \ell}^{++} & =\frac{M_{V^{+}+} M_{\ell}^{2}\left(\tilde{g}^{(1)}\right)^{2}}{96 \pi \Lambda^{2}}\left[1-4 x_{\ell}^{2}\right]^{3 / 2}, \\
\Gamma_{2, \ell}^{++} & =\frac{M_{\mathcal{V}^{+}+\left(\tilde{g}^{(2)}\right)^{2}}}{24 \pi}\left[1-4 x_{\ell}^{2}\right]^{3 / 2}, \\
\Gamma_{3, \ell}^{++} & =\frac{M_{\mathbf{V}^{++}} M_{\ell}^{2}\left(\tilde{g}^{(3)}\right)^{2}}{96 \pi \Lambda^{2}}\left[1-4 x_{\ell}^{2}\right]^{3 / 2}, \\
\Gamma_{2, \ell}^{+} & =\frac{M_{\mathcal{V}^{+}}\left(\tilde{g}^{(2)}\right)^{2}}{48 \pi}\left[2-3 x_{\ell}^{2}+x_{\ell}^{6}\right], \\
\Gamma_{3, \ell}^{+} & =\frac{M_{\mathbf{V}^{+}} M_{\ell}^{2}\left(\tilde{g}^{(3)}\right)^{2}}{384 \pi \Lambda^{2}}\left[1-x_{\ell}^{2}\right]^{2}\left[2+x_{\ell}^{2}\right] .
\end{aligned}
$$

As in the previous sections, flavor violating decays have not been considered as the underlying interactions could lead to visible signals in rare leptonic decay experiments.

\section{MULTILEPTON PRODUCTION AT THE LHC}

In this section, we present numerical predictions of total cross sections for the production of multileptonic final states originating from the pair production and decay of the various new particles introduced in Section 2 For the sake of simplicity, we assume that all new physics masses are equal. We focus on $p p$ collisions such as produced at the CERN LHC collider running at a center-of-mass energy $\sqrt{S_{h}}=8 \mathrm{TeV}$. Thanks to the QCD factorization theorem, hadronic cross sections are calculated by convolving the partonic cross sections $\hat{\sigma}$ computed in Section 2 with the universal parton densities $f_{a}$ and $f_{b}$ of partons $a$ and $b$ in the proton, which depend on the longitudinal momentum fractions of the two partons $x_{a, b}=\sqrt{\tau} e^{ \pm y}$ and on the unphysical factorization scale $\mu_{F}$,

$$
\sigma=\int_{4 \tilde{M}^{2} / S_{h}}^{1} \mathrm{~d} \tau \int_{-1 / 2 \ln \tau}^{1 / 2 \ln \tau} \mathrm{d} y f_{a}\left(x_{a}, \mu_{F}^{2}\right) f_{b}\left(x_{b}, \mu_{F}^{2}\right) \hat{\sigma}\left(x_{a} x_{b} S_{h}\right) .
$$



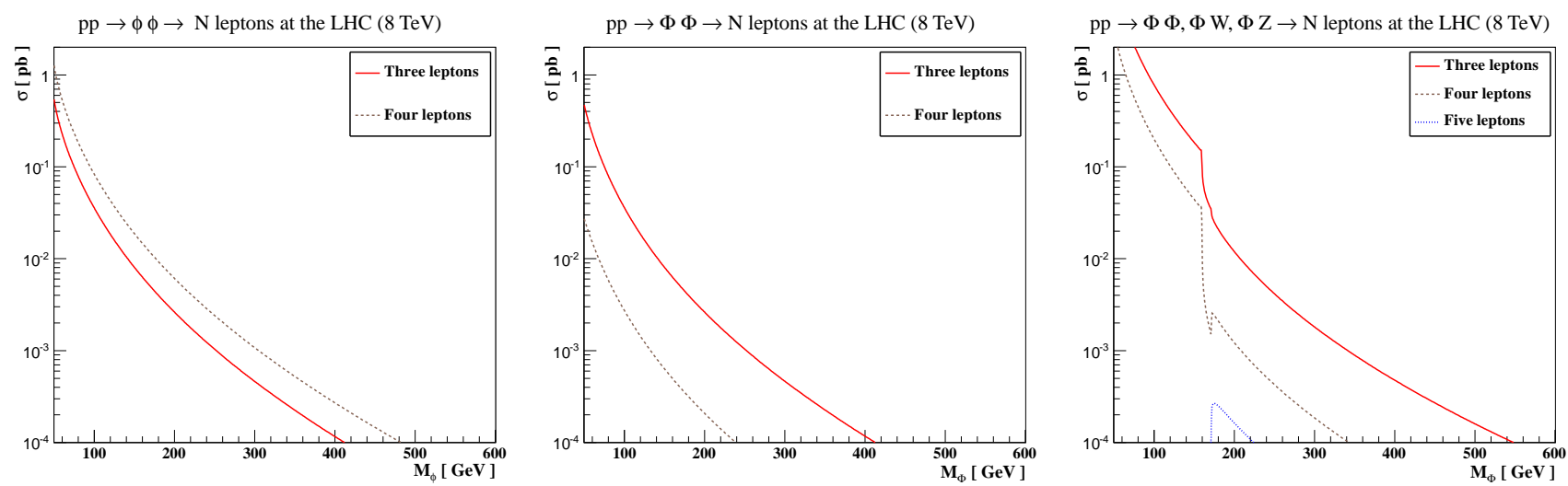

FIG. 1: Doubly-charged particle contributions to the production rate of final states containing more than three charged leptons at the LHC, running at a center-of-mass energy of $8 \mathrm{TeV}$. We consider non-standard fields lying in the singlet (left), doublet (center) and triplet (right) representations of $S U(2)_{L}$ and impose that its component with the highest electric charge to be doubly-charged.

We employ the leading order set L1 of the CTEQ6 global parton density fit [88], which includes five light quark flavors and the gluon and we identify the factorization scale to be the average mass of the produced final state particles $\tilde{M}$.

For the masses and widths of the electroweak gauge bosons, we use the current values given in the Particle Data Group review [89], i.e., $M_{Z}=91.1876 \mathrm{GeV}, M_{W}=80.385 \mathrm{GeV}, \Gamma_{Z}=2.4952 \mathrm{GeV}$ and $\Gamma_{W}=2.085 \mathrm{GeV}$, and we evaluate the CKM-matrix elements by using the Wolfenstein parameterization. The corresponding four free parameters are set to $\lambda=0.22535, A=0.811, \bar{\rho}=0.131$ and $\bar{\eta}=0.345$. The squared sine of the electroweak mixing angle $\sin ^{2} \theta_{W}=1-M_{W}^{2} / M_{Z}^{2}$ and the electromagnetic fine structure constant $\alpha=\sqrt{2} G_{F} M_{W}^{2} \sin ^{2} \theta_{W} / \pi$ are calculated in the improved Born approximation using a value of $G_{F}=1.16638 \cdot 10^{-5} \mathrm{GeV}^{-2}$ for the Fermi coupling constant, which is derived from muon lifetime measurements.

We start by focusing on mechanisms yielding the production of pairs of components of the $S U(2)_{L}$ multiplets $\phi, \Phi$ and $\boldsymbol{\Phi}$ introduced in Section 2.1. In the first step, we evaluate the different branching ratios of their doubly-charged, singly-charged and neutral states to a given number of electrons, muons and taus by using Eq (2.12), Eq. (2.13), Eq. (2.14) and Eq. (2.15). We then derive, in Figure 1, BSM contributions to the production rates of final states containing $N_{\ell}=3, N_{\ell}=4$ and $N_{\ell}=5$ charged leptons ${ }^{2}$ originating from the production and decay of the new scalar particles after accounting for subsequent decays of tau leptons, $W$-bosons and $Z$-bosons where relevant. For our numerical analysis, we choose to couple the $\phi, \Phi$ and $\boldsymbol{\Phi}$ fields to charged leptons in a flavor-conserving way, as already mentioned in the previous section of this paper, and we fix the parameters of the Lagrangian of Eq. (2.6) to

$$
y^{(1)}=y^{(2)}=y^{(3)}=0.1 \cdot \mathbb{1} .
$$

Moreover, we consider the effective interactions of the doublet fields to be suppressed by an energy scale $\Lambda=1 \mathrm{TeV}$ and allow the neutral component of the triplet field $\boldsymbol{\Phi}^{0}$ to acquire a non-vanishing vacuum expectation value equal to $v_{\boldsymbol{\Phi}}=100 \mathrm{GeV}$. Finally, all new particles are assumed mass-degenerate.

On the left panel of the figure, we present results for a field lying in the singlet representation of $S U(2)_{L}$. The unique component of such a field, i.e., the doubly charged particle $\phi^{++}$, always decays into a charged lepton pair or a tau pair. Four lepton signatures are therefore expected to be copiously produced, although the possible hadronic decays of the tau allow for contributions to final states containing less than four electrons and muons. Consequently, doubly-charged particles lying in the singlet representation of $S U(2)_{L}$ contribute, for moderate masses $M_{\phi} \lesssim 330 \mathrm{GeV}$, to the production of final states with a leptonic multiplicity $N_{\ell} \geq 3$, with a cross section larger than $1 \mathrm{fb}$.

The situation changes when the Standard Model is extended by fields lying in a non-trivial representation of $S U(2)_{L}$ due to the possibility of production of associated pairs of different new states or of a single new state together with a Standard Model gauge boson. This is illustrated on the central and right panels of Figure 1 for the doublet and triplet cases, respectively. For fields lying in the fundamental representation of $S U(2)_{L}$, the BSM contributions to

\footnotetext{
${ }^{2}$ From now on, we denote by the terminology charged leptons, electrons and muons.
} 

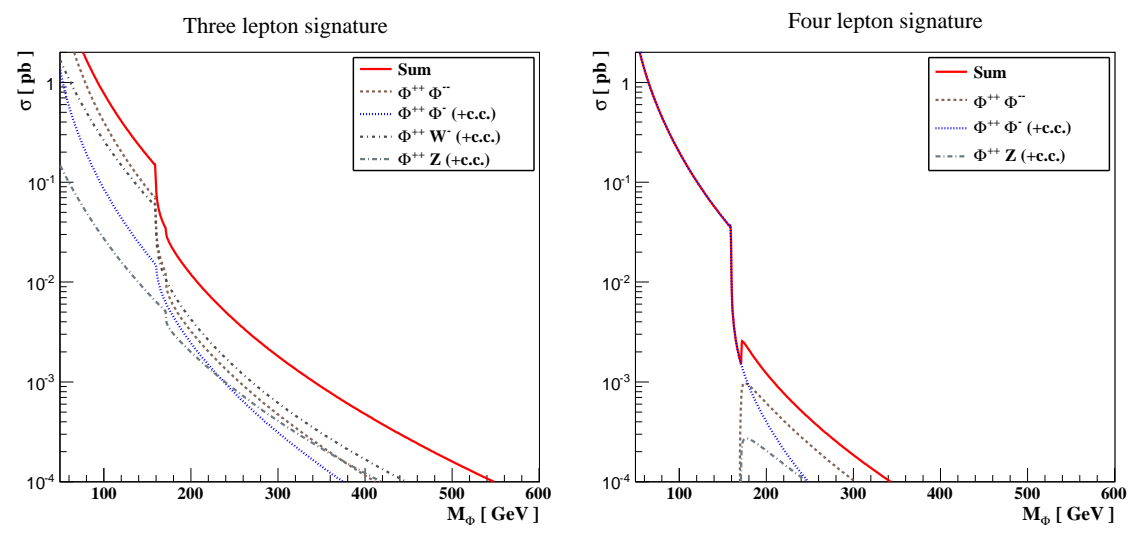

FIG. 2: Different new physics contributions to the production of three (left) and four (right) leptons at the LHC, running at a center-of-mass energy of $8 \mathrm{TeV}$, in the context of a simplified model containing one additional $S U(2)_{L}$ triplet of scalar fields whose highest component is a doubly-charged state.
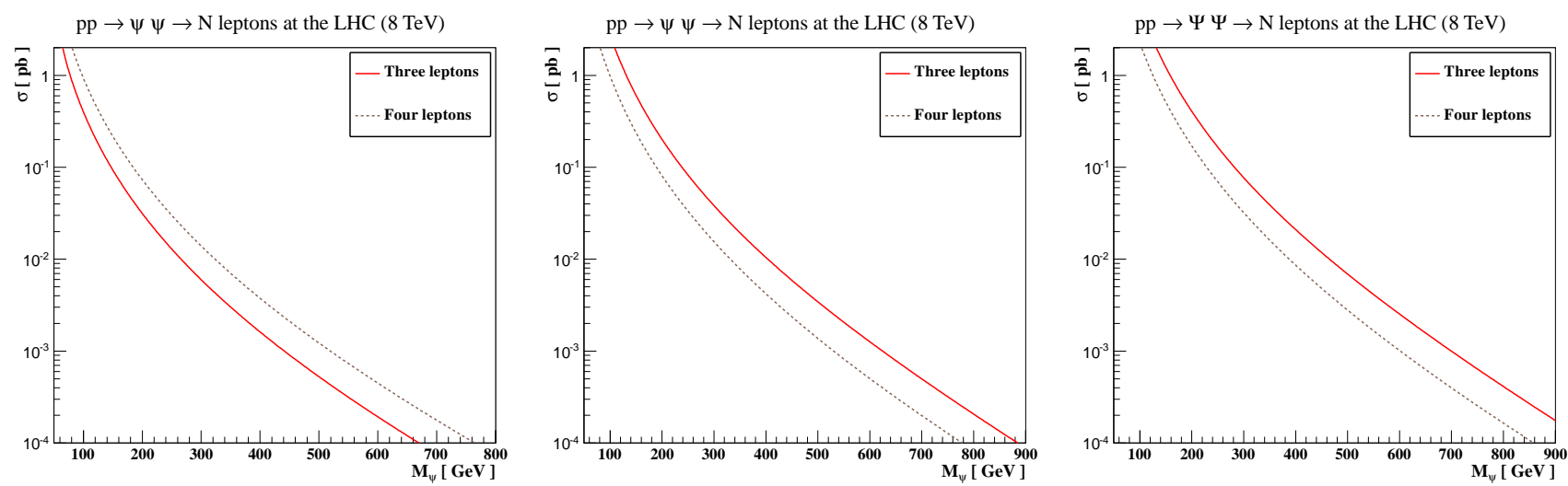

FIG. 3: Doubly-charged particle production rate of final states containing more than three charged leptons at the LHC, running at a center-of-mass energy of $8 \mathrm{TeV}$. We consider extensions of the Standard Model with an extra fermionic field lying in the singlet (left), doublet (center) or triplet (right) representation of $S U(2)_{L}$ and impose that its component with the highest electric charge to be doubly-charged. In addition, its singly-charged component is prevented from mixing with the SM sector.

the production cross section of final states with $N_{\ell} \geq 3$ charged leptons are found to be larger than 1 fb only for a rather low mass scale $M_{\Phi} \lesssim 250 \mathrm{GeV}$. For fields in the adjoint representation, the predictions however highly depend on the size of the vev $v_{\boldsymbol{\Phi}}$. For very small $v_{\boldsymbol{\Phi}}$ values, the cross sections are sizable and a large mass reach can be foreseen, as extrapolated from the results presented in the region of the right panel of Figure 1 located below the dibosonic thresholds, where the value of the vev is irrelevant. However, as soon as the dibosonic decay modes of the $\boldsymbol{\Phi}^{+}$and $\boldsymbol{\Phi}^{++}$fields are open, important vev values render them dominant so that the production rate of final states with more than three charged leptons drops, the Standard Model weak bosons preferably decaying into quarks. In our example, where the vev has a value close to the weak scale, the new physics masses have to be below $350 \mathrm{GeV}$ in order to contribute to the production cross sections of multileptonic final states (with $N_{\ell} \geq 3$ ) with at least $\sigma=1 \mathrm{fb}$. We show in detail the effects of a large $v_{\Phi}$ in Figure 2, where we split the different production channels contributing to final state signatures with $N_{\ell}=3$ (left panel) and $N_{\ell}=4$ (right panel) charged leptons and present the respective contribution of each subprocess.

In addition, it should be noted that final states with five and six leptons can also be produced, since both the doubly-charged and singly-charged fields can decay into three leptons after accounting for leptonic $W$-boson and $Z$ boson decays. However, the related rates are very low and render the possible observation of five-lepton or six-lepton events rather unlikely when one takes into account the available integrated luminosity of $20 \mathrm{fb}^{-1}$ recorded at the LHC in 2012 .

We now turn to the pair-production of the components of the fermionic fields $\psi, \Psi$ and $\boldsymbol{\Psi}$ introduced in Section 2.2 

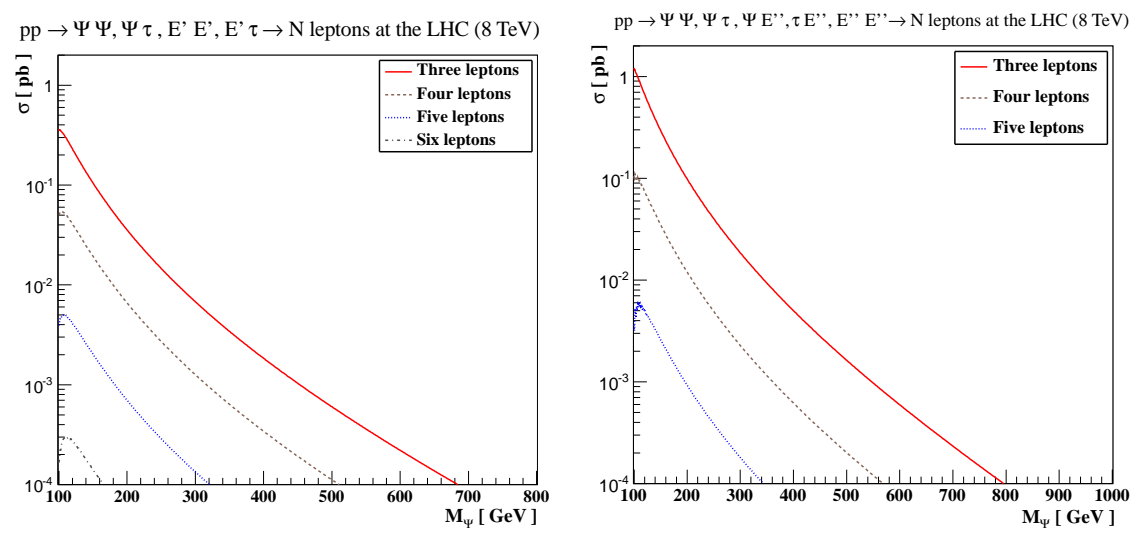

FIG. 4: Doubly-charged particle production rate of final states containing more than three charged leptons at the LHC, running at a center-of-mass energy of $8 \mathrm{TeV}$. We consider extensions of the Standard Model with an extra fermionic field lying in the singlet (left), doublet (center) or triplet (right) representation of $S U(2)_{L}$ and impose its component with the highest electric charge ti be doubly-charged. In addition, its singly-charged component mixes with the SM tau lepton.

and calculate their effect on the production of final states containing three leptons or more at the LHC. We investigate first scenarios with three generations of fermions as presented in Section 2.2.1. In this case, the new states decay through a four-fermion interaction into a pair of leptons with the same electric charge and a fermionic field $N$ similar to a right-handed neutrino, as described by Eq. (2.18). In order to compute the related partial widths, we implement the Lagrangian of Eq. (2.18) into the FEYNRules package 90 95], then export the Feynman rules to a UFO module [96] that is subsequently linked to MADGRAPH 5 [97]. For our numerical analysis, we set the effective scale $\Lambda=1 \mathrm{TeV}$ and impose the coupling strengths to be flavor-diagonal,

$$
G^{(1,1)}=G^{(1,2)}=G^{(2,1)}=G^{(2,2)}=G^{(3,1)}=G^{(3,2)}=0.1 \cdot \mathbb{1} .
$$

We also set the mass of the $N$-field to $50 \mathrm{GeV}$, in order to be compatible with the current limits on the existence of heavy stable neutral leptons [89] and assume, for the sake of simplicity, that all components of the new fermionic multiplet containing the doubly-charged state have the same mass. In Figure 3, we derive the associated contributions to the production of final states with at least three charged leptons in the singlet (left panel), doublet (central panel) and triplet (right panel) cases. In contrast to scalar extra fields, we observe that a much larger mass range is expected to give rise to cross sections larger than $1 \mathrm{fb}$, which is guaranteed for $M_{\psi} \lesssim 550 \mathrm{GeV}, M_{\Psi} \lesssim 650 \mathrm{GeV}$ and $M_{\Psi} \lesssim 725 \mathrm{GeV}$ for new fermionic fields lying in the singlet, doublet and triplet representations of $S U(2)_{L}$, respectively.

In the series of fermionic scenarios presented in Section 2.2.2 the Standard Model is only supplemented by a fermionic field lying either in the fundamental or in the adjoint representation of $S U(2)_{L}$. In this case, the singlycharged component mixes with the Standard Model tau lepton, which allows for the decays of the new states to the Standard Model sector and open new production mechanisms giving rise to signatures with $N_{\ell} \geq 3$ charged leptons. In order not to challenge the very precisely measured properties of the tau lepton inferred by its coupling to the $Z$-boson, we fix the mixing angle

$$
\sin \theta_{\tau}=0.01
$$

Computing the different branching ratios using the formulas of Eq. (2.22), Eq. (2.23) and Eq. (2.26) we then show in Figure 4 the new physics contributions to the production of final states with at least three leptons. It is found that for masses $M_{\Psi} \lesssim 470 \mathrm{GeV}$ and $M_{\Psi} \lesssim 550 \mathrm{GeV}$ in the doublet and triplet cases, respectively, the corresponding cross sections are higher than $1 \mathrm{fb}$.

We finally address the production of the vectorial multiplets $V, \mathcal{V}$ and $\mathbf{V}$ defined in Section 2.3 These fields lie in the trivial, fundamental and adjoint representation of $S U(2)_{L}$, respectively, and their component with the highest electric charge is doubly-charged. In order to derive the associated contributions to the production of signatures with three leptons and more, we start by computing the different partial widths of the new fields according to the results of Eq. (2.33). As for the other types of models, we choose the coupling strengths of the new field to the Standard Model leptons and neutrinos, presented in Eq.(2.30), to be flavor-conserving,

$$
\tilde{g}^{(1)}=\tilde{g}^{(1)}=\tilde{g}^{(1)}=0.1 \cdot \mathbb{1},
$$



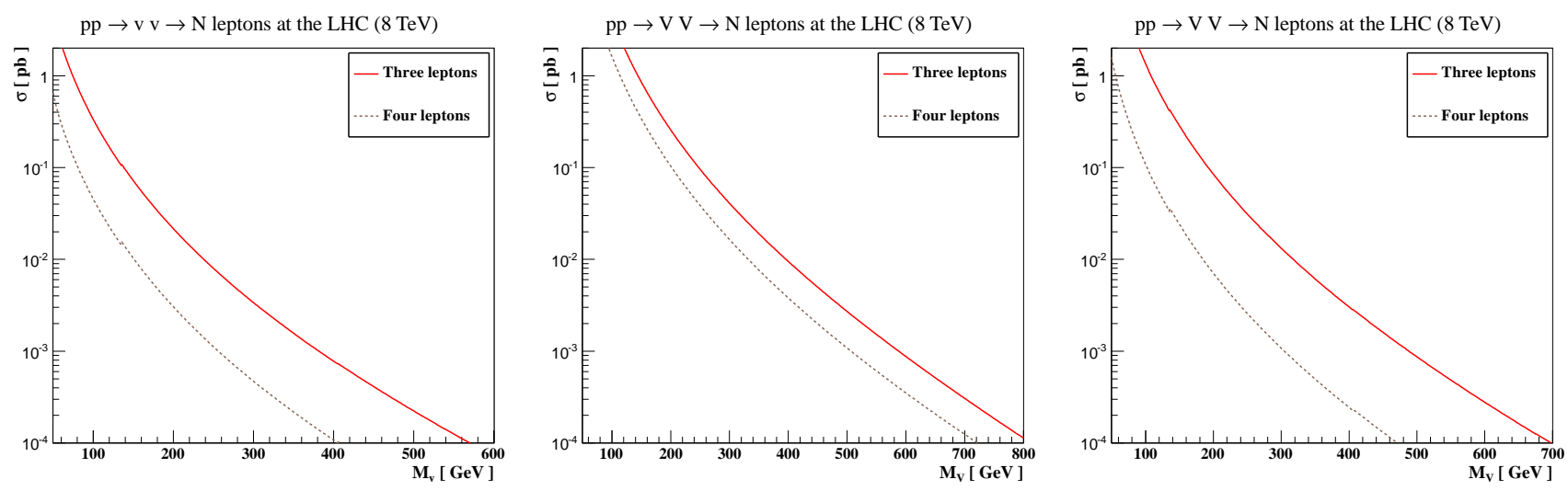

FIG. 5: Doubly-charged particle production rate of final states containing more than three charged leptons at the LHC, running at a center-of-mass energy of $8 \mathrm{TeV}$. We consider extensions of the Standard Model with an extra vectorial field lying in the singlet (left), doublet (center) or triplet (right) representation of $S U(2)_{L}$ and impose that its component with the highest electric charge to be doubly-charged.

TABLE I: Upper bound on doubly-charged particle mass scale so that the sum of all BSM contributions to the production rate, at the LHC running at a center-of-mass energy of $8 \mathrm{TeV}$, of multileptonic final states (with $N_{\ell} \geq 3$ ) is larger than $1 \mathrm{fb}$.

\begin{tabular}{l||c|c|c} 
& $S U(2)_{L}$ singlet & $S U(2)_{L}$ doublet & $S U(2)_{L}$ triplet \\
\hline Scalar fields & $330 \mathrm{GeV}$ & $257 \mathrm{GeV}$ & $350 \mathrm{GeV}$ \\
Fermionic fields (no mixing with the SM) & $555 \mathrm{GeV}$ & $661 \mathrm{GeV}$ & $738 \mathrm{GeV}$ \\
Fermionic fields (mixing with the SM) & - & $471 \mathrm{GeV}$ & $560 \mathrm{GeV}$ \\
Vector fields & $392 \mathrm{GeV}$ & $619 \mathrm{GeV}$ & $495 \mathrm{GeV}$
\end{tabular}

and suppressed, if relevant, by an effective scale fixed to $\Lambda=1 \mathrm{TeV}$. Moreover, all new states are once again assumed mass-degenerate. We show in Figure 5 the hadronic cross sections related to trilepton and tetralepton production induced by the pair-production of the component of the $V, \mathcal{V}$ and $\mathbf{V}$ fields. We find that these cross sections are larger than $1 \mathrm{fb}$, which possibly implies the observation of some events during the 2012 LHC run, for new physics masses satisfying $M_{V} \lesssim 400 \mathrm{GeV}, M_{\mathcal{V}} \lesssim 600 \mathrm{GeV}$ and $M_{\mathbf{V}} \lesssim 500 \mathrm{GeV}$ in the singlet, doublet and triple cases, respectively.

In Table \. we summarize the different mass ranges expected to give rise to new physics contributions to multilepton production at the LHC collider, running at a center-of-mass energy of $8 \mathrm{TeV}$, larger than $1 \mathrm{fb}$. This motivates us to select three benchmark scenarios for a more careful analysis, based on Monte Carlo simulations, of the effects associated with the presence of fields containing a doubly-charged component, with the aim of defining some ways to distinguish their spin and $S U(2)_{L}$ representation. We hence choose first a series of scenarios where the common mass is fixed to a rather optimistic value of $100 \mathrm{GeV}$, recalling that no LHC constraints has been derived for promptly decaying fields and/or when non-leptonic decay channels are open. We then define two other classes of scenarios where the new physics mass scale lies well above the dibosonic thresholds. We fix it to $250 \mathrm{GeV}$ and $350 \mathrm{GeV}$, respectively.

\section{PROBING SPIN AND $S U(2)_{L}$ REPRESENTATIONS WITH MONTE CARLO SIMULATIONS}

In the previous section, we have shown that the mass range for possibly observing doubly-charged particles at the LHC depends on their spin and $S U(2)_{L}$ representations. In this section, we focus on the study of various kinematical distributions that should allow to probe the nature of a doubly-charged particle, if one assumes that it is responsible for the observation of excesses in multilepton final states with $N_{\ell} \geq 3$ charged leptons. Towards this goal, we implement all the models presented in Section 2 in MadGraph 5 [97] via FeynRules [90 95]. We then present, within the MADANALYSIS 5 framework [98], results that are based on a hadron-level simulation of the signal describing $20 \mathrm{fb}^{-1}$ of collisions at a center-of-mass energy of $8 \mathrm{TeV}$. For this, the parton-level events as generated by MADGRAPH 5 have been showered and hadronized by means of the Pүтнін 6 package [99], tau lepton decays being handled by using the TAUOLA program [100]. 
We start our analysis by preselecting events after imposing a set of basic selections ensuring that most (nonsimulated) background contributions are well under control.

- We start by removing from the event final states all charged leptons not having a transverse momentum $p_{T} \geq 10 \mathrm{GeV}$ and a pseudorapidity satisfying $|\eta| \leq 2.5$.

- Jets are reconstructed by means of the anti- $k_{t}$ algorithm [101] as implemented in FASTJET [102, 103] after we set the radius parameter to $R=0.4$. We only consider jet candidates with $p_{T} \geq 20 \mathrm{GeV}$ and $|\eta| \leq 2.5$ which are not too close to an electron, i.e., which lie outside a cone of radius $R=0.1$ centered around the electron.

- Lepton isolation is then enforced by rejecting all leptons lying in a cone of radius $R=0.4$ centered on any of the remaining jets.

- We require the presence in the final state of at least three isolated charged leptons.

While a complete simulation of the Standard Model background goes beyond the scope of this work, we refer to an existing phenomenological study of leptonic final states to demonstrate that the background remaining after the selection above ${ }^{3}$ is under control [104]. This analysis shows that we can indeed expect about 5500 background events, originating in $99.5 \%$ of the cases from diboson production processes, so that already 230 signal events can induce a $3 \sigma$ deviation from the Standard Model expectation.

Since all experimental analyses focusing on multileptonic signatures further select events by requiring specific thresholds on the transverse-momentum of (at least) the two leading leptons $\ell_{1}$ and $\ell_{2}$, we present the related spectra in Figure 6 and Figure 7 in the context of all the Standard Model extensions introduced in Section 2 In the top, middle and lower series of graphs shown on the figures, respectively, we focus on fields lying in the singlet, doublet and triplet representations of $S U(2)_{L}$. In contrast, on the left, central and right columns of the figure, we set the masses of the new states to $100 \mathrm{GeV}, 250 \mathrm{GeV}$ and $350 \mathrm{GeV}$, respectively. We recall that these choices have been adopted from the three mass scenarios constructed in the previous section. All the represented spectra exhibit a common global behavior. The distributions start by steeply rising, then peak and are finally extended by a tail up to (in general) moderate $p_{T}$ values smaller than $400 \mathrm{GeV}$. It is therefore rather complicated to define a feature to a given spin and/or $S U(2)_{L}$ representation when one accounts for the possible different new particle masses. Two exceptions are however allowed. First, events containing very hard leptons with a transverse momentum larger than $500 \mathrm{GeV}$ are expected to be copiously produced in scenarios where the Standard Model is extended by a vectorial field $\mathcal{V}$ lying in the doublet representation of $S U(2)_{L}$, for any mass value. Next, models with additional doubly-charged scalar fields that are singlet under the $S U(2)_{L}$ gauge group lead to the production of multileptonic events where the $p_{T}$ spectra of the two leading leptons are depleted in the low and intermediate transverse-momentum regions. This feature nevertheless competes with the low cross sections associated with heavy scalar masses larger than 250-300 GeV (as illustrated in Figure 11).

From those considerations, one concludes that the $p_{T}$ spectra of the two leading leptons $\ell_{1}$ and $\ell_{2}$ offer possible means to distinguish the spin and/or $S U(2)_{L}$ representations of the doubly-charged particles in very specific cases, but not in general. Similar features are found when analyzing the transverse-momentum distribution of the next-tonext-to-leading lepton $\ell_{3}$, as well as the transverse-mass and the invariant-mass spectra of any pair of leptons present in the event. The corresponding figures have therefore been omitted for brevity.

Spin representations are highly correlated to angular distributions. In this way, scalar, fermionic and vectorial doubly-charged particles are expected to give rise to signals with largely different features, when investigating kinematical variables such as angular distances between final state particles. As an example, in Figure 8 we show the distribution in the angular distance between the two leading leptons $\Delta R\left(\ell_{1} \ell_{2}\right)=\sqrt{\Delta \phi_{12}^{2}+\Delta \eta_{12}^{2}}$. In this expression, $\Delta \phi_{12}$ stands for the azimuthal angular separation of the two leptons with respect to the beam direction and $\Delta \eta_{12}$ for their pseudorapidity difference. Investigating the shapes of the spectra, we observe that they not only depends on the Lorentz representation of the new field, but also on their $S U(2)_{L}$ one. Therefore, these variables offer an important discriminating features among the different scenarios and deserve to be studied in the context of a more realistic phenomenological analysis, including detector effects that could alter the spectrum. As we wish to keep our considerations as general as possible, this however is beyond the scope of this prospective work.

\footnotetext{
${ }^{3}$ Although an additional veto on events containing identified $b$-jets is applied, this does not affect our purely leptonic signal.
} 
$p p \rightarrow N_{\ell}$ charged leptons at the LHC $(8 \mathrm{TeV})$, with $N_{\ell} \geq 3$.
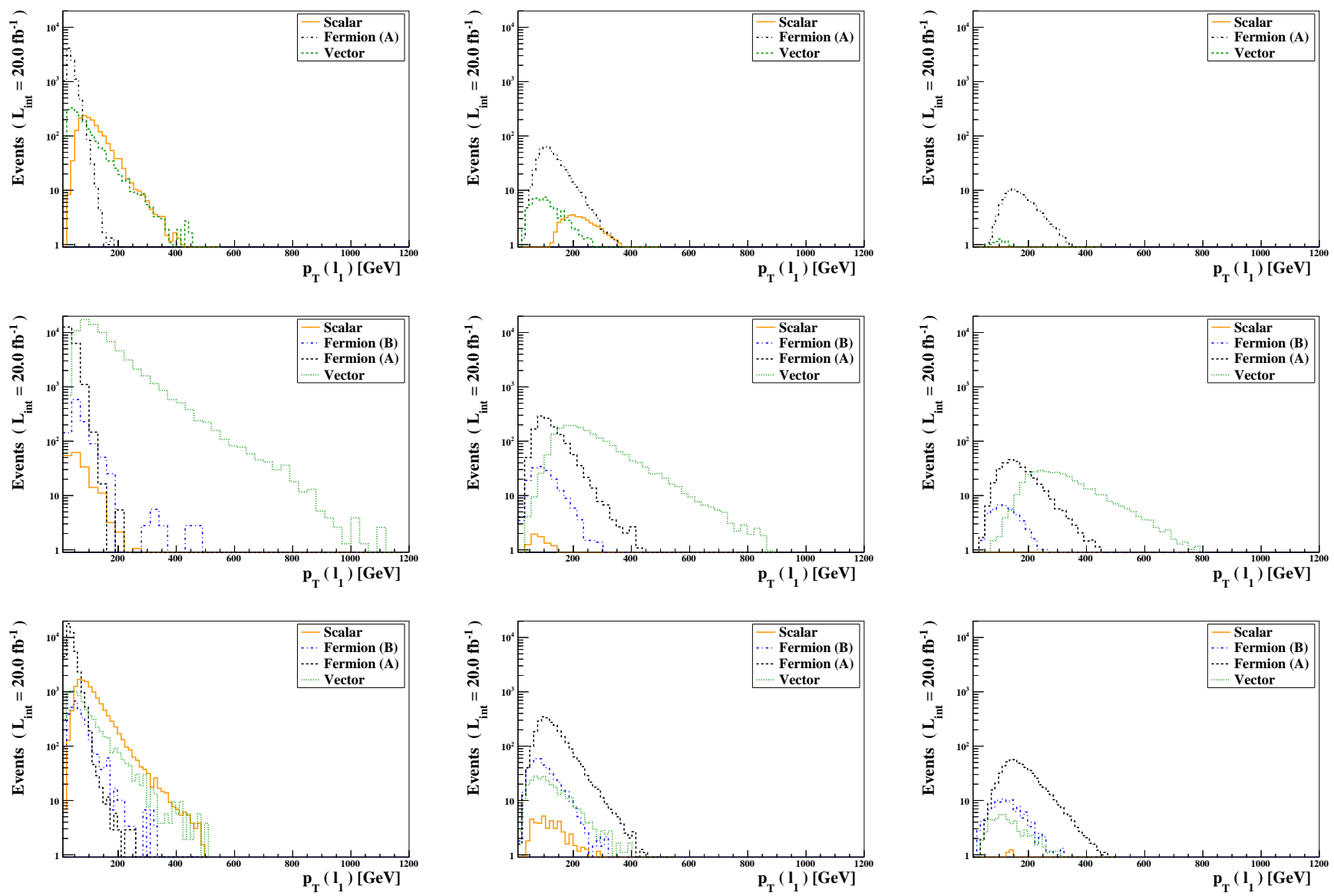

FIG. 6: Transverse-momentum spectrum of the leading lepton emerging from a possible doubly-charged particle signal with $N_{\ell} \geq 3$ charged leptons in the final states. Event generation has been performed in the context of the LHC and for $20 \mathrm{fb}^{-1}$ of collisions at a center-of-mass energy of $8 \mathrm{TeV}$. In the top, middle and lower series of graphs, we require that the new states lie in the trivial, fundamental and adjoint representation of $S U(2)_{L}$, respectively, while their mass is set to $100 \mathrm{GeV}$, $250 \mathrm{GeV}$ and $350 \mathrm{GeV}$ in the left, central and right columns of the figure. In each subfigure, we show distributions for scalar fields (plain orange curve), vector fields (dashed green curve) and fermionic fields whose singly-charged component is allowed to mix with the Standard Model $\tau$ lepton (dashed blue curve, dubbed scenario B) or not (dot-dashed black curve, dubbed scenario A).

\section{CONCLUSIONS}

In this paper we have investigated LHC signals related to the presence of doubly-charged particles. We have considered different scenarios for such particles, varying their Lorentz and $S U(2)_{L}$ representations and constructing associated simplified models allowing for their pair production, followed by their decays into Standard Model particles. We have studied the contributions of doubly-charged states to the production cross sections of final states containing three or more charged leptons, a signature known to suffer from a reduced Standard Model background. Using analytical and numerical computations we have hence deduced that masses ranging up to $700 \mathrm{GeV}$ are possibly accessible at the LHC for specific models. We have then employed Monte Carlo simulations to probe several kinematical distributions allowing to possibly distinguish the spin and $S U(2)_{L}$ representations of a doubly-charged state. The results found have been encouraging, in particular in the case of variables such as the angular distance $\Delta R$ between two of the final state leptons. This motivates an extension of this work, including an investigation of the detector effects which could spoil the shapes of the angular variable spectra, possibly in the context of ATLAS or CMS analyses. 
$p p \rightarrow N_{\ell}$ charged leptons at the LHC $(8 \mathrm{TeV})$, with $N_{\ell} \geq 3$.
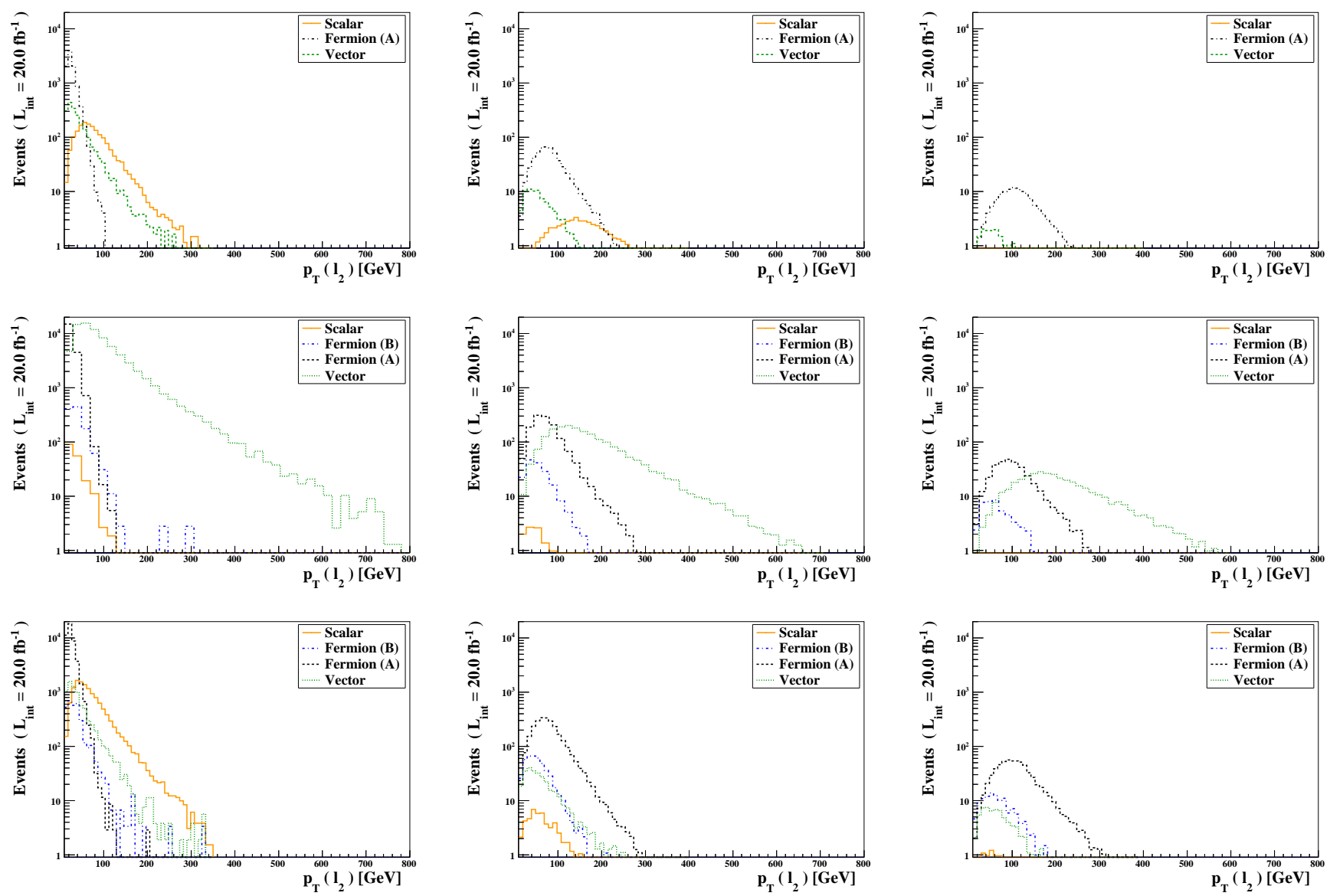

FIG. 7: Same as in Figure 6] but for the transverse-momentum spectrum of the next-to-leading leading charged lepton.

\section{Acknowledgments}

The authors are grateful to Claude Duhr and Olivier Mattelaer for discussing fermion number violating interactions in MADGRAPH. We acknowledge NSERC of Canada for partial financial support under grant number SAP105354 and the Theory-LHC-France initiative of the CNRS/IN2P3.

[1] G. Aad et al. [ATLAS Collaboration], Phys. Lett. B 710 (2012) 49.

[2] S. Chatrchyan et al. [CMS Collaboration], Phys. Lett. B 710 (2012) 26.

[3] F. Cuypers and S. Davidson, Eur. Phys. J. C 2 (1998) 503.

[4] E. Del Nobile, R. Franceschini, D. Pappadopulo and A. Strumia, Nucl. Phys. B 826 (2010) 217.

[5] V. Rentala, W. Shepherd and S. Su, Phys. Rev. D 84 (2011) 035004.

[6] B. Meirose and A. A. Nepomuceno, Phys. Rev. D 84 (2011) 055002.

[7] J. Hisano and K. Tsumura, arXiv:1301.6455 [hep-ph].

[8] F. del Aguila, M. Chala, A. Santamaria and J. Wudka, arXiv:1305.3904 [hep-ph].

[9] J. C. Pati and A. Salam, Phys. Rev. D 10 (1974) 275i [Erratum-ibid. D 11 (1975) 703].

[10] R. N. Mohapatra and J. C. Pati, Phys. Rev. D 11 (1975) 566.

[11] R. N. Mohapatra and J. C. Pati, Phys. Rev. D 11 (1975) 2558.

[12] G. Senjanovic and R. N. Mohapatra, Phys. Rev. D 12 (1975) 1502. 
$p p \rightarrow N_{\ell}$ charged leptons at the LHC $(8 \mathrm{TeV})$, with $N_{\ell} \geq 3$.
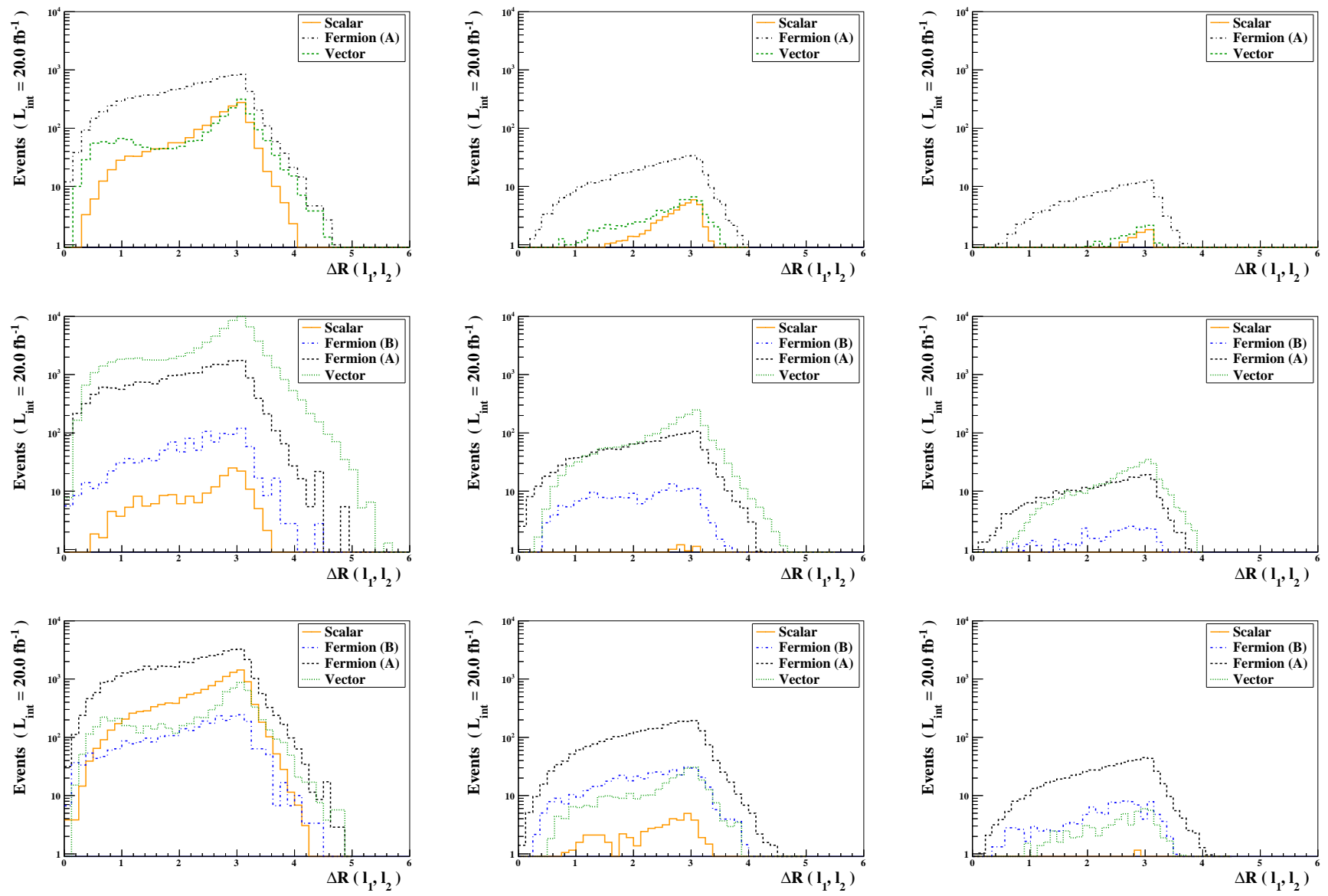

FIG. 8: Same as in Figure 6, but for the angular-distance spectrum of a lepton pair comprised of the two leading leptons $\ell_{1}$ and lepton $\ell_{2}$.

[13] R. N. Mohapatra, F. E. Paige and D. P. Sidhu, Phys. Rev. D 17 (1978) 2462.

[14] G. Senjanovic, Nucl. Phys. B 153 (1979) 334.

[15] R. N. Mohapatra and G. Senjanovic, Phys. Rev. Lett. 44 (1980) 912.

[16] T. P. Cheng and L. -F. Li, Phys. Rev. D 22 (1980) 2860.

[17] G. B. Gelmini and M. Roncadelli, Phys. Lett. B 99 (1981) 411.

[18] A. Zee, Phys. Lett. B 93 (1980) 389 [Erratum-ibid. B 95 (1980) 461].

[19] T. Han, H. E. Logan, B. Mukhopadhyaya and R. Srikanth, Phys. Rev. D 72 (2005) 053007.

[20] J. Y. Lee, JHEP 0506 (2005) 060.

[21] I. Picek and B. Radovcic, Phys. Lett. B 687 (2010) 338.

[22] S. K. Majee and N. Sahu, Phys. Rev. D 82 (2010) 053007.

[23] M. Aoki, S. Kanemura and K. Yagyu, Phys. Lett. B 702 (2011) 355 [Erratum-ibid. B 706 (2012) 495].

[24] M. -C. Chen and J. Huang, Mod. Phys. Lett. A 26 (2011) 1147.

[25] K. Kumericki, I. Picek and B. Radovcic, Phys. Rev. D 84 (2011) 093002.

[26] M. Aoki, S. Kanemura and K. Yagyu, Phys. Rev. D 85 (2012) 055007.

[27] C. -W. Chiang, T. Nomura and K. Tsumura, Phys. Rev. D 85 (2012) 095023.

[28] K. Kumericki, I. Picek and B. Radovcic, Phys. Rev. D 86 (2012) 013006.

[29] H. Sugiyama, K. Tsumura and H. Yokoya, Phys. Lett. B 717 (2012) 229.

[30] I. Picek and B. Radovcic, Phys. Lett. B 719 (2013) 404.

[31] S. Kanemura, K. Yagyu and H. Yokoya, arXiv:1305.2383 [hep-ph]. 
[32] C. Csaki, C. Delaunay, C. Grojean and Y. Grossman, JHEP 0810 (2008) 055.

[33] M. -C. Chen, K. T. Mahanthappa and F. Yu, Phys. Rev. D 81 (2010) 036004.

[34] A. Kadosh and E. Pallante, JHEP 1008 (2010) 115.

[35] F. del Aguila, A. Carmona and J. Santiago, JHEP 1008 (2010) 127.

[36] F. del Aguila, A. Carmona and J. Santiago, Phys. Lett. B 695 (2011) 449.

[37] A. Delgado, C. Garcia Cely, T. Han and Z. Wang, Phys. Rev. D 84 (2011) 073007.

[38] M. Cvetic, J. Halverson and P. Langacker, JHEP 1111 (2011) 058

[39] D. A. Demir, M. Frank, K. Huitu, S. K. Rai and I. Turan, Phys. Rev. D 78 (2008) 035013.

[40] M. Frank, K. Huitu and S. K. Rai, Phys. Rev. D 77 (2008) 015006.

[41] K. S. Babu, A. Patra and S. K. Rai, arXiv:1306.2066 [hep-ph].

[42] R. Franceschini and R. N. Mohapatra, arXiv:1306.6108 [hep-ph].

[43] P. H. Frampton and B. -H. Lee, Phys. Rev. Lett. 64 (1990) 619.

[44] P. B. Pal, Phys. Rev. D 43 (1991) 236.

[45] F. Pisano and V. Pleitez, Phys. Rev. D 46 (1992) 410.

[46] P. H. Frampton, Mod. Phys. Lett. A 7 (1992) 2017.

[47] P. H. Frampton, Phys. Rev. Lett. 69 (1992) 2889.

[48] E. Farhi and L. Susskind, Phys. Rept. 74 (1981) 277.

[49] H. Harari, Phys. Rept. 104 (1984) 159.

[50] N. Cabibbo, L. Maiani and Y. Srivastava, Phys. Lett. B 139 (1984) 459.

[51] E. Eichten, I. Hinchliffe, K. D. Lane and C. Quigg, Rev. Mod. Phys. 56 (1984) 579 [Addendum-ibid. 58 (1986) 1065].

[52] G. Pancheri and Y. N. Srivastava, Phys. Lett. B 146 (1984) 87.

[53] W. Buchmuller, Acta Phys. Austriaca Suppl. 27 (1985) 517.

[54] C. A. Stephan, J. Phys. A 39 (2006) 9657.

[55] S. B. Gudnason, C. Kouvaris and F. Sannino, Phys. Rev. D 73 (2006) 115003.

[56] S. Biondini, O. Panella, G. Pancheri, Y. N. Srivastava and L. Fano, Phys. Rev. D 85 (2012) 095018.

[57] G. Abbiendi et al. [OPAL Collaboration], Phys. Lett. B 526 (2002) 221.

[58] G. Abbiendi et al. [OPAL Collaboration], Phys. Lett. B 577 (2003) 93.

[59] P. Achard et al. [L3 Collaboration], Phys. Lett. B 576 (2003) 18.

[60] J. Abdallah et al. [DELPHI Collaboration], Phys. Lett. B 552 (2003) 127.

[61] J. Sztuk-Dambietz [H1 and ZEUS Collaboration], J. Phys. Conf. Ser. 110 (2008) 072042.

[62] A. Aktas et al. [H1 Collaboration], Phys. Lett. B 638 (2006) 432.

[63] V. M. Abazov et al. [D0 Collaboration], Phys. Rev. Lett. 101 (2008) 071803.

[64] V. M. Abazov et al. [D0 Collaboration], Phys. Rev. Lett. 108 (2012) 021801.

[65] D. E. Acosta et al. [CDF Collaboration], Phys. Rev. Lett. 93 (2004) 221802.

[66] T. Aaltonen et al. [The CDF Collaboration], Phys. Rev. Lett. 101 (2008) 121801.

[67] S. Chatrchyan et al. [ CMS Collaboration], arXiv:1305.0491 [hep-ex].

[68] G. Aad et al. [ ATLAS Collaboration], Phys. Lett. B 722 (2013) 305.

[69] S. Chatrchyan et al. [CMS Collaboration], Eur. Phys. J. C 72 (2012) 2189.

[70] G. Aad et al. [ATLAS Collaboration], Eur. Phys. J. C 72 (2012) 2244.

[71] D. Alves et al. [LHC New Physics Working Group Collaboration], arXiv:1105.2838 [hep-ph].

[72] J. F. Gunion, J. Grifols, A. Mendez, B. Kayser and F. I. Olness, Phys. Rev. D 40 (1989) 1546.

[73] C. Csaki, J. Hubisz, G. D. Kribs, P. Meade and J. Terning, Phys. Rev. D 67 (2003) 115002.

[74] M. -C. Chen and S. Dawson, Phys. Rev. D 70 (2004) 015003.

[75] C. Csaki, J. Hubisz, G. D. Kribs, P. Meade and J. Terning, Phys. Rev. D 68 (2003) 035009.

[76] T. Rommerskirchen and T. Hebbeker, J. Phys. G G 34 (2007) N47.

[77] A. G. Akeroyd and M. Aoki, Phys. Rev. D 72 (2005) 035011.

[78] A. G. Akeroyd, M. A. Diaz, J. Ferrandis, M. A. Garcia-Jareno and J. W. F. Valle, Nucl. Phys. B 529 (1998) 3.

[79] M. L. Swartz, Phys. Rev. D 40 (1989) 1521.

[80] T. G. Rizzo, Phys. Rev. D 25 (1982) 1355 [Addendum-ibid. D 27 (1983) 657]. 
[81] J. F. Gunion, J. Grifols, A. Mendez, B. Kayser and F. I. Olness, Phys. Rev. D 40 (1989) 1546.

[82] T. S. Kosmas, G. K. Leontaris and J. D. Vergados, Prog. Part. Nucl. Phys. 33 (1994) 397.

[83] R. N. Mohapatra, Phys. Rev. D 46 (1992) 2990.

[84] S. F. King, Rept. Prog. Phys. 67 (2004) 107.

[85] G. Altarelli and F. Feruglio, New J. Phys. 6 (2004) 106.

[86] T. Asaka, S. Blanchet and M. Shaposhnikov, Phys. Lett. B 631 (2005) 151.

[87] M. Frank, Phys. Rev. D 62 (2000) 053004.

[88] J. Pumplin, D. R. Stump, J. Huston, H. L. Lai, P. M. Nadolsky and W. K. Tung, JHEP 0207 (2002) 012.

[89] J. Beringer et al. [Particle Data Group Collaboration], Phys. Rev. D 86 (2012) 010001.

[90] N. D. Christensen and C. Duhr, Comput. Phys. Commun. 180 (2009) 1614.

[91] N. D. Christensen, P. de Aquino, C. Degrande, C. Duhr, B. Fuks, M. Herquet, F. Maltoni and S. Schumann, Eur. Phys. J. C 71 (2011) 1541.

[92] N. D. Christensen, C. Duhr, B. Fuks, J. Reuter and C. Speckner, Eur. Phys. J. C 72 (2012) 1990.

[93] C. Duhr and B. Fuks, Comput. Phys. Commun. 182 (2011) 2404.

[94] B. Fuks, Int. J. Mod. Phys. A 27 (2012) 1230007.

[95] A. Alloul, J. D'Hondt, K. De Causmaecker, B. Fuks and M. Rausch de Traubenberg, Eur. Phys. J. C 73 (2013) 2325.

[96] C. Degrande, C. Duhr, B. Fuks, D. Grellscheid, O. Mattelaer and T. Reiter, Comput. Phys. Commun. 183 (2012) 1201.

[97] J. Alwall, M. Herquet, F. Maltoni, O. Mattelaer and T. Stelzer, JHEP 1106 (2011) 128.

[98] E. Conte, B. Fuks and G. Serret, Comput. Phys. Commun. 184 (2013) 222.

[99] T. Sjostrand, S. Mrenna and P. Z. Skands, JHEP 0605 (2006) 026.

[100] N. Davidson, G. Nanava, T. Przedzinski, E. Richter-Was and Z. Was, Comput. Phys. Commun. 183 (2012) 821.

[101] M. Cacciari, G. P. Salam and G. Soyez, JHEP 0804 (2008) 063.

[102] M. Cacciari and G. P. Salam, Phys. Lett. B 641 (2006) 57.

[103] M. Cacciari, G. P. Salam and G. Soyez, Eur. Phys. J. C 72 (2012) 1896.

[104] A. Alloul, M. Frank, B. Fuks and M. Rausch de Traubenberg, arXiv:1307.5073 [hep-ph]. 